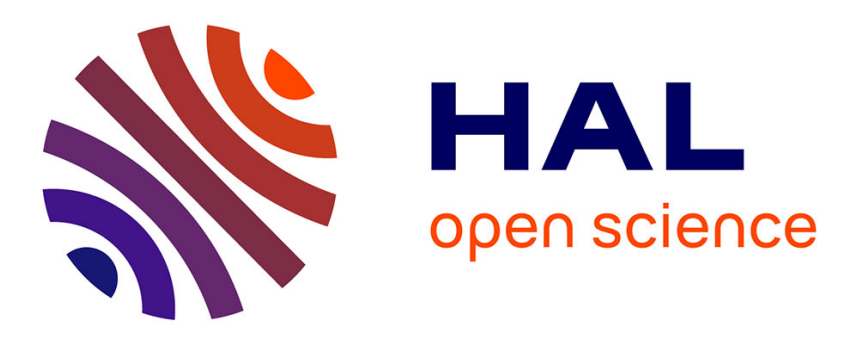

\title{
Sur les ions lents dans une chambre de simulation du plasma ionosphérique
}

A. Soubeyran, L. Lévy, D. Sarrail, E. Coggiola, Jean-Jacques Berthelier

\section{To cite this version:}

A. Soubeyran, L. Lévy, D. Sarrail, E. Coggiola, Jean-Jacques Berthelier. Sur les ions lents dans une chambre de simulation du plasma ionosphérique. Revue de Physique Appliquée, 1990, 25 (11), pp.1177-1195. 10.1051/rphysap:0199000250110117700 . jpa-00246287

\section{HAL Id: jpa-00246287 https://hal.science/jpa-00246287}

Submitted on 1 Jan 1990

HAL is a multi-disciplinary open access archive for the deposit and dissemination of scientific research documents, whether they are published or not. The documents may come from teaching and research institutions in France or abroad, or from public or private research centers.
L'archive ouverte pluridisciplinaire HAL, est destinée au dépôt et à la diffusion de documents scientifiques de niveau recherche, publiés ou non, émanant des établissements d'enseignement et de recherche français ou étrangers, des laboratoires publics ou privés. 
Classification

Physics Abstracts

$52.30-52.40 \mathrm{~K}-\mathrm{M}-52.65-94.20$

\title{
Sur les ions lents dans une chambre de simulation du plasma ionosphérique
}

\author{
A. Soubeyran $\left({ }^{1}\right)$, L. Lévy $\left({ }^{1}\right)$, D. Sarrail $\left({ }^{1}\right)$, E. Coggiola $\left({ }^{2}\right)$ et J. J. Berthelier $\left({ }^{3}\right)$ \\ (') Département d'Etude et de Recherche en Technologie Spatiale, Centre d'Etude et de Recherche de \\ Toulouse, 2 avenue E. Belin, 31055 Toulouse cedex, France \\ (2) \# 245, Petrie Science Building, Physics Department, North York, Ontario, M3J 1P3, Canada \\ ( ${ }^{3}$ ) Centre de Recherche en Physique de l'Environnement (CNET/CNRS), 4 avenue de Neptune, 94107 Saint \\ Maur des Fossés, France
}

(Reçu le 10 avril 1990, révisé le 2 juillet 1990, accepté le 12 juillet 1990)

\begin{abstract}
Résumé. - Nous établissons une formulation analytique de la densité des ions lents en fonction de paramètres expérimentaux pertinents, et appliquons avec succès cette expression à l'interprétation de résultats de mesures. Par ailleurs, nous décrivons la simulation numérique que nous avons développée pour appréhender les phénomènes physiques intervenant en présence d'un sillage électrostatique.
\end{abstract}

\begin{abstract}
An analytical formulation of the cold ions density is established, which exhibit the influence of the main facility parameters. This expression is succesfully applied to explain experimental results. A numerical simulation is developped which authorized a better comprehension of the migration and collection of cold ions in the near-wake of a satellite mock-up.
\end{abstract}

\section{Introduction.}

Pour décrire l'interaction entre un satellite et le plasma ionosphérique, plusieurs approches sont envisageables. La plus directe consiste à réaliser des mesures in situ, en cours de vol. En pratique, on se heurte rapidement à des problèmes d'interprétation dans un environnement très complexe, où les capteurs sont rarement dans des conditions de fonctionnement optimal. Par ailleurs cette méthode est particulièrement onéreuse.

Ainsi vient l'idée d'expériences à échelle réduite, dans des chambres à plasma adéquates, où les conditions sont mieux contrôlées. On place une un courant de lasma et l'on peut alors étudier finement l'interaction. Depuis vingt cinq ans, cette méthode a fait l'objet de nombreuses investigations fructueuses $[19,18,20$, 9]. Cependant, le domaine de variation des paramètres utilisables ne permet pas une stricte analogie avec le milieu ionosphérique et certains phénomènes parasites peuvent affecter la validité des résultats. Il en est ainsi de la présence d'un plasma d'ions lents, produits par échange de charge entre les ions dérivants rapidement et la population neutre résiduelle $[7,6,10]$.
Ce plasma froid peut avoir une influence plus ou moins sensible sur les mesures, suivant sa densité et le type d'expériences menées. D'une façon générale, les expérimentateurs s'accordent souvent pour négliger cette influence si sa densité ne dépasse pas quelques pour-cent de celle du plasma dérivant [12]. Cependant, cette proportion est souvent difficile à mesurer directement, et il n'existe à notre connaissance, que peu de recherches relatives à ses variations avec les paramètres expérimentaux $[1,4,13]$. Nous avons donc décidé d'approfondir ce problème en développant deux approches complémentaires, dans les conditions simplifiées d'absence de champ

La première méthode consiste en une formulation analytique, partiellement empirique, des relations entre les divers paramètres choisis pour caractériser le système. Cette approche simplifiée n'est valable que dans un domaine limité, où elle a cependant l'avantage d'offrir des résultats quantitatifs et des tendances directement comparables aux mesures. Par contre, les phénomènes physiques dont elle représente un modèle, ne sont pas toujours explicites. 
L'autre méthode consiste en une simulation numérique de l'interaction d'un objet polarisé de géométrie simple, avec un plasma dérivant, en présence d'un plasma froid. C'est un peu l'analogue spatial des "souffleries numériques" de l'aéronautique. Plus encore que ces dernières, elle souffre des limitations de géométries et de modèles physiques réducteurs, nécessaires pour permettre des calculs en un temps compatible avec les capacités des ordinateurs disponibles (FPS 64, et bientôt CRAY XMP). Cependant, les paramètres de l'interaction sont ici parfaitement maîtrisés, les diagnostiques peuvent être nombreux, sans perturbations pour le système, et les conditions d'interactions peuvent donc être connues de façon plus fines que par l'approche analytique. Ainsi, la compréhension qualitative des phénomènes physiques en jeu est alors généralement accessible. Même si les résultats quantitatifs ne sont pas toujours directement comparables aux données expérimentales, nous avons là un instrument privilégié de liaison entre les conditions en vol et les expérimentations au sol.
Enfin, nous avons comparé les résultats de ces approches avec deux ensembles de données expérimentales indépendants. D'une part, à l'occasion d'une étude sur les problèmes de charge et de sillage en orbite basse, réalisée dans la grande chambre à plasma de "Physikalisch-Technische Studien" (PTS) à Freiburg, nous mettons en évidence le rôle prépondérant du plasma froid dans les mesures [5]. D'autre part, nous constatons un très bon accord avec des résultats expérimentaux de Pigache et Fournier obtenus dans la chambre à plasma de I'ONERA [7].

\section{Le calcul analytique.}

Dans un premier temps, nous recherchons la densité moyenne des ions lents, supposée uniforme. Nous verrons que, moyennant certaines- hypothèses, cette densité peut être reliée au courant collecté sur une sonde de mesure. Nous appliquerons ensuite ce modèle aux mesures obtenues dans les chambres à plasma de PTS et de l'ONERA.
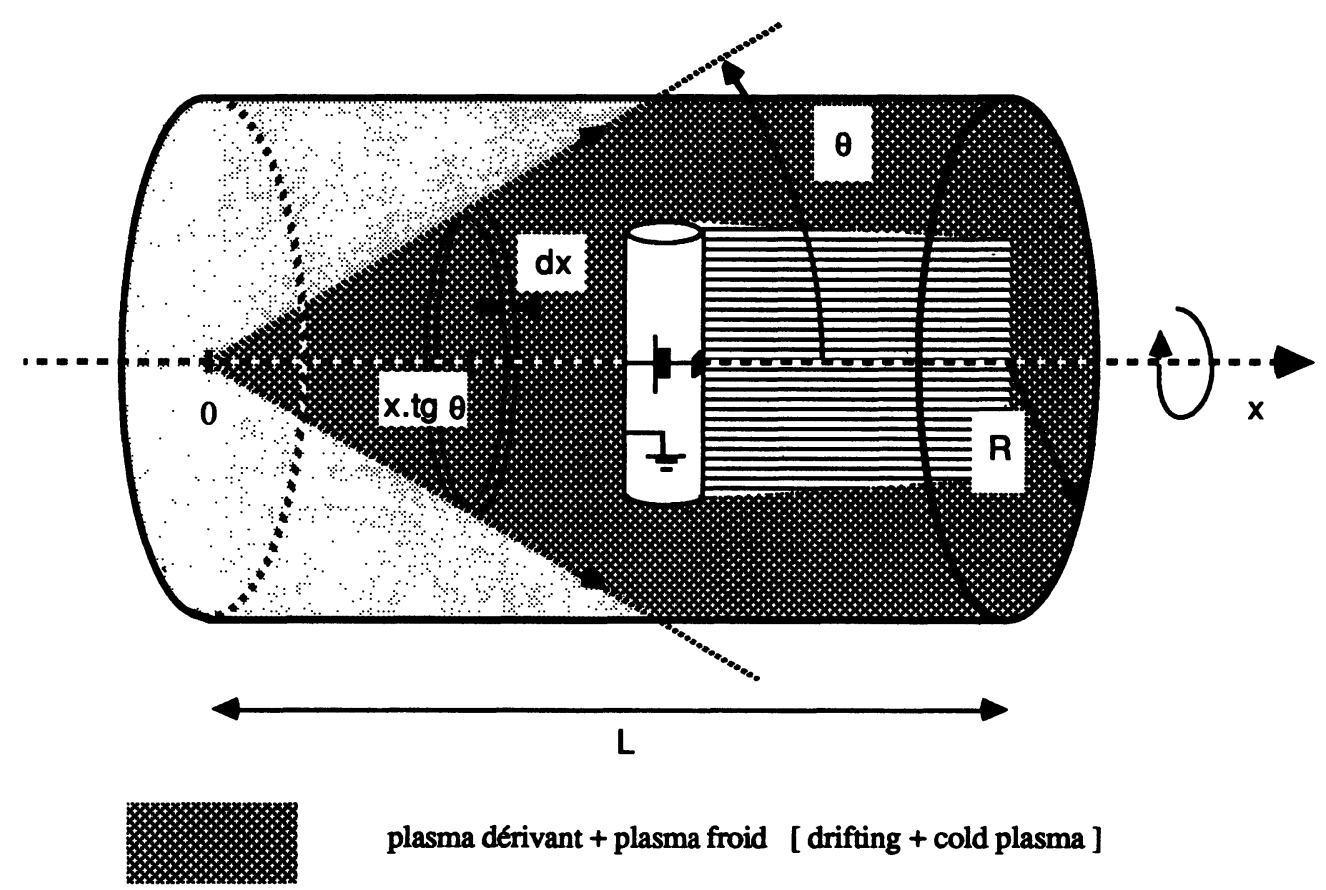

plasma dérivant + plasma froid [ drifting + cold plasma ]

plasma froid [cold plasma]

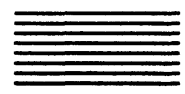

sillage [wake]

Fig. 1. - Schéma de la chambre à plasma de PTS, où l'on a représenté l'axe de symétrie $x$, et l'angle d'ouverture du faisceau de plasma dérivant $\theta$. La maquette de mesures cylindrique est figurée au centre, avec sa surface polarisée négativement dans le sillage.

[Schematic representation of the PTS plasma chamber, with the revolution axis $x$ and the plasma beam aperture angle $\theta$. The instrumented mock-up stay in the middle of the chamber, with a negatively polarised surface in the wake.] 
2.1 DENSITÉ DES IONS LENTS. - La figure 1 représente schématiquement une chambre à plasma telle que celle utilisée à PTS. Il s'agit d'une cuve cylindrique de rayon $R$, de longueur $L$, chacun de l'ordre de quelques mètres, de surface $S$ et de volume $V$. On y distingue le faisceau de plasma dérivant émis par une

- Ions dérivants

densité :

vitesse de dérive :

- Ions lents

densité moyenne :

nombre :

vitesse de dérive :

température :

- Electrons

densité : température :

- Plasma hors de la zone perturbée :

densité :

longueur de Debye :

libre parcours moyen ion-ion:

libre parcours moyen ion-neutre :

- Atomes neutres

densité uniforme :

température :

Compte tenu de ses conditions de production et de disparition, le plasma froid n'est pas uniforme en toute rigueur. C'est pourquoi nous parlerons de densité moyenne d'ions lents. Le calcul qui suit est fondé sur l'égalité en régime stationnaire, entre la source volumique $Q_{\mathrm{il}}$ et la perte surfacique $L_{\mathrm{il}}$ de ces ions.

2.1.1 Le terme de source. - On fait l'hypothèse très largement admise [1, 12, 4, 13], que les ions lents sont produits majoritairement par la réaction d'échange de charge résonnant :

$$
\mathrm{Ar}_{\mathrm{d}}^{+}+\mathrm{Ar}_{1} \rightarrow \mathrm{Ar}_{\mathrm{d}}+\mathrm{Ar}_{1}^{+} \text {. }
$$

L'espèce chimique est l'argon, les indices $\mathrm{d}$ et 1 font référence à «dérivant » et "lent». Cette réaction correspond, en première approximation, au transfert d'un électron, sans échange de quantité de mouvemen . a sec ion e icace sera no ee: $\sigma$.

$\mathrm{La}$ dispersion de vitesses des ions dérivants est supposée faible et, dans le domaine de vitesses étudié, la variation de la section efficace est très petite, inférieure aux incertitudes sur sa valeur (Fig. 2) [14]. Le taux de production, dépendant de $x$ par l'intermédiaire de $n_{\text {id }}$ s'écrira donc [14]:

$$
\tau(x)=n_{\mathrm{n}} \cdot n_{\mathrm{id}}(x) \cdot \sigma \cdot v_{\mathrm{id}} .
$$

La densité $n_{\text {id }}(x)$ se calcule aisément en considérant la «tranche » d'épaisseur $\mathrm{d} x$ représentée sur la source située en $O$, ainsi que le plasma d'ions lents distribué uniformément. La maquette instrumentée cylindrique était placée verticalement, vers le milieu de la chambre, comme indiqué sur la figure 1.

Les principaux paramètres caractéristiques du contenu sont :

$\begin{array}{ll}\begin{array}{ll}n_{\text {id }}(x) \\ v_{\text {id }}\end{array} & 10^{11}-10^{12} \mathrm{~m}^{-3} \\ & \text { quelques } 10^{4} \mathrm{~m} \cdot \mathrm{s}^{-1} \\ n_{\mathrm{il}} & \\ N_{\mathrm{il}}=n_{\mathrm{il}} \cdot V & 10^{10}-10^{12} \mathrm{~m}^{-3} \\ 0 & \\ T_{\mathrm{il}} & \approx T_{\mathrm{n}} \\ & \\ n_{\mathrm{e}} & 10^{11}-10^{12} \mathrm{~m}^{-3} \\ T_{\mathrm{e}} & \approx 0,1 \mathrm{eV} \\ & \\ n_{\mathrm{il}}+n_{\text {id }} & =n_{\mathrm{e}} \\ \lambda_{\mathrm{D}} & 10^{-2}-10^{-3} \mathrm{~m} \\ & 5 \times 10^{2}-10^{3} \mathrm{~m} \\ & 2,5-10^{3} \mathrm{~m} \\ n_{\mathrm{n}} & 10^{16}-10^{18} \mathrm{~m}^{-3} \\ T_{\mathrm{n}} & \approx 300 \mathrm{~K}^{2}\end{array}$

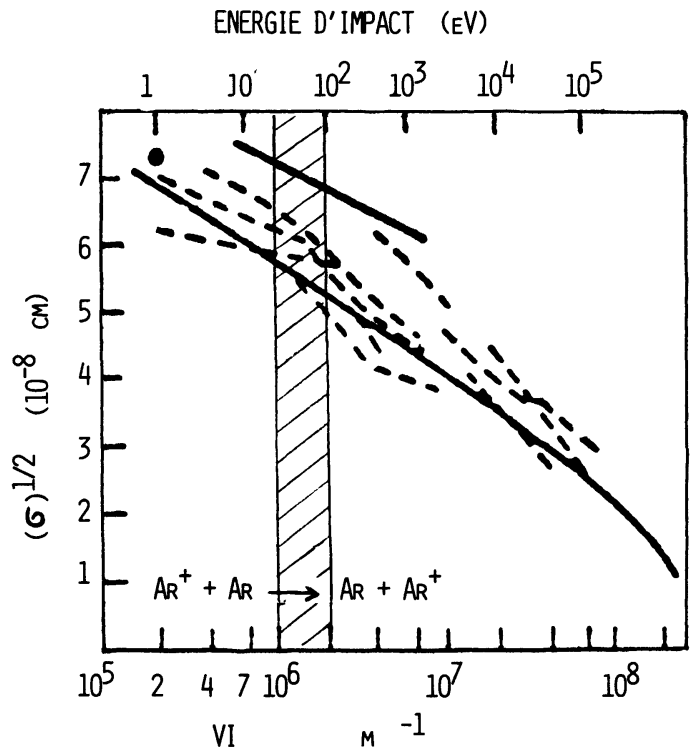

Fig. 2. - Section efficace d'échange de charge pour l'argon, en fonction de l'énergie ou de la vitesse de l'ion primaire. Les courbes pleines correspondent aux résultats théoriques, les courbes pointillées, aux résultats expérimentaux (d'après [14]). Notre domaine d'étude se trouve dans la partie hachurée.

[Resonance charge transfer cross section versus impact velocity. Broken lines indicate experimental data, and full lines indicate calculations (after [14]). The dashed region indicates our working area.] 
figure 1. Lors de son passage dans cette tranche, la densité d'ions dérivants va diminuer, d'une part par transformation en ions lents, d'autre part en raison de la dispersion géométrique dans le cône d'éjection :

$$
\begin{aligned}
\frac{\partial n_{\mathrm{id}}(x)}{\partial x}=-\frac{\tau}{v_{\mathrm{id}}} & -n_{\mathrm{id}}(x) \times \frac{2}{x} \\
\rightarrow \text { ions lents } & \text { dispersion géométrique }
\end{aligned}
$$

En supposant que l'on mesure $\left[n_{\text {id }}\left(L_{\mathrm{s}}\right)=n_{\text {id }}\right]$ par une sonde située à la distance $L_{\mathrm{s}}$ de la source de plasma et compte tenu de (1), on en déduit :

$$
n_{\mathrm{id}}(x)=n_{\mathrm{id}} \times\left(\frac{L_{\mathrm{s}}}{x}\right)^{2} \times \mathrm{e}^{-n_{\mathrm{n}} \times \sigma \times\left(x-L_{\mathrm{s}}\right)} .
$$

On admet que les ions disparaissent par neutralisation lors de leur impact sur la paroi métallique de la chambre. Le taux d'émission secondaire ou de diffusion est en effet très faible à ces énergies $(\approx 0,03 \mathrm{eV})$.

La production d'ions lents s'écrit alors :

$$
\begin{aligned}
Q_{\mathrm{il}}=\int_{v} \tau & \times \mathrm{d} V=\int_{v}\left[n_{\mathrm{n}} \times \sigma \times v_{\mathrm{id}} \times n_{\mathrm{id}} \times\right. \\
& \left.\times\left(\frac{L_{\mathrm{s}}}{x}\right)^{2} \times \mathrm{e}^{-n_{\mathrm{n}} \times \sigma \times\left(x-L_{\mathrm{s}}\right)}\right] \times \mathrm{d} V .
\end{aligned}
$$

$\tau$ ne dépendant que de $x$, nous allons prendre comme élément de volume $\mathrm{d} V$ une tranche d'épaisseur $\mathrm{d} x$. Cependant, en fonction de $x$, le rayon de cette tranche croit jusqu'à $R$, celui de la chambre, et s'y maintient ensuite. L'intégrale doit donc être généralement scindée en deux parties :

$$
\begin{aligned}
Q_{\mathrm{il}}= & n_{\mathrm{n}} \times n_{\mathrm{id}} \times \sigma \times v_{\mathrm{id}} \times\left(L_{\mathrm{s}}\right)^{2} \times \mathrm{e}^{n_{\mathrm{n}} \times \sigma \times L_{\mathrm{s}}} \times \\
& \times\left[\int_{0}^{\frac{R}{\operatorname{tg} \theta}} \frac{\mathrm{e}^{-n_{\mathrm{n}} \times \sigma \times x}}{\mathrm{x}^{2}} \times \pi \times(x \times \operatorname{tg} \theta)^{2}\right. \\
& \left.\times \mathrm{d} x+\int_{\frac{R}{\operatorname{tg} \theta}}^{L} \frac{e^{-n_{\mathrm{n}} \times \sigma \times x}}{x^{2}} \times \pi \times R^{2} \times \mathrm{d} x\right] .
\end{aligned}
$$

Si la première intégrale ne pose pas de problèmes, par contre la seconde ne se résoud pas simplement. Bien que sa primitive puissent s'écrire sous forme d'un développement en série, une telle écriture n'est pas d'un emploi aisé, en particulier en vue d'une étude paramétrique en fonction de $n_{\mathrm{n}}$. Aussi avons nous préféré ramener cette intégrale à une forme semblable à la première. L'intégrale cherchée est majorée par le prolongement de la première intégrale entre $R / \operatorname{tg} \theta$ et $L$. En effet, l'élément de volume $y$ est plus petit, du fait que les ions dérivants ayant atteint la paroi ne peuvent plus engendrer d'ions lents. Supposons qu'il existe une distance
$L_{\mathrm{f}}$ (longueur effective) comprise entre $R / \operatorname{tg} \theta$ et $L$, telle que :

$$
\begin{aligned}
& \int_{\frac{R}{\operatorname{tg} \theta}}^{L} \frac{\mathrm{e}^{-n_{\mathrm{n}} \times \sigma \times x}}{x^{2}} \times \pi \times R^{2} \times \mathrm{d} x= \\
& \quad=\int_{\frac{R}{\operatorname{tg} \theta}}^{L_{\mathrm{f}}} \frac{\mathrm{e}^{-n_{\mathrm{n}} \times \sigma \times x}}{x^{2}} \times \pi \times(x \times \operatorname{tg} \theta)^{2} \times \mathrm{d} x .
\end{aligned}
$$

Bien entendu, cette idée n'est intéressante que dans la mesure où $L_{\mathrm{f}}$ est indépendante de la variable expérimentale $n_{\mathrm{n}}$. Nous avons vérifié par le calcul numérique d'intégrales dans les cas extrêmes que tel était le cas, à $5 \%$ près dans tout le domaine de valeurs pertinentes pour notre étude. Ainsi on peut écrire :

$$
\begin{aligned}
& \int_{0}^{\frac{R}{\operatorname{tg} \theta}} \frac{\mathrm{e}^{-n_{\mathrm{n}} \times \sigma \times x}}{x^{2}} \times \pi \times(x \times \operatorname{tg} \theta)^{2} \times \mathrm{d} x+ \\
& +\int_{\frac{R}{\operatorname{tg} \theta}}^{L} \frac{\mathrm{e}^{-n_{\mathrm{n}} \times \sigma \times x}}{x^{2}} \times \pi \times R^{2} \times \mathrm{d} x \approx \pi \times(\operatorname{tg} \theta)^{2} \\
& \times \int_{0}^{L_{\mathrm{f}}} \frac{\mathrm{e}^{-n_{\mathrm{n}} \times \sigma \times x}}{x^{2}} \times \mathrm{d} x=\frac{\pi \times(\operatorname{tg} \theta)^{2}}{\sigma \times n_{\mathrm{n}}} \times \\
& \times\left(1-\mathrm{e}^{-n_{\mathrm{n}} \times \sigma \times L_{\mathrm{f}}}\right)
\end{aligned}
$$

et :

$$
\begin{aligned}
Q_{\mathrm{il}} \approx n_{\mathrm{id}} \times v_{\mathrm{id}} & \times \pi \times\left(L_{\mathrm{s}} \times \operatorname{tg} \theta\right)^{2} \times \\
& \times\left(1-\mathrm{e}^{-n_{\mathrm{n}} \times \sigma \times L_{\mathrm{f}}}\right) \times \mathrm{e}^{-n_{\mathrm{n}} \times \sigma \times L_{\mathrm{s}}} .
\end{aligned}
$$

2.1.2 Le terme de perte. - Nous négligerons les pertes sur la maquette, dont la surface de collection est très petite devant celle de la chambre, même en présence d'une polarisation modérée, comme le montrent les simulations numériques. Le flux des ions lents à la paroi est égal à celui entrant dans la gaine, dont l'ordre de grandeur peut être estimé à l'aide du critère de Bohm [3] :

$$
F \approx F_{\mathrm{B} \mathrm{ohm}} \approx 0,6 \times n_{\mathrm{il}} \times \sqrt{\frac{k \times T_{\mathrm{e}}}{m_{\mathrm{i}}}}
$$

où $m_{\mathrm{i}}$ est la masse des ions et $k$ la constante de Boltzmann.

Cette formule n'est valable en toute rigueur que pour une géométrie plane et une température ionique nulle.

Finalement :

$L_{\mathrm{il}} \approx F \times S \approx 3,8 \times n_{\mathrm{il}} \times \sqrt{\frac{k \times T_{\mathrm{e}}}{m_{\mathrm{i}}}} \times R \times(R+L)$.

2.1.3 Régime stationnaire. - On écrit l'égalité des sources (6) et des pertes (7) :

$$
Q_{\mathrm{il}}=L_{\mathrm{il}} \text {. }
$$


D'où l'on déduit le rapport des densités d'ions lents et d'ions dérivants, au point de mesure de la sonde $L_{\mathrm{s}}$ :

$$
\begin{aligned}
& \frac{n_{\mathrm{il}}}{n_{\mathrm{id}}} \approx 0,82 \times \frac{\left(L_{\mathrm{s}} \times \operatorname{tg} \theta\right)^{2}}{R \times(R+L)} \times v_{\mathrm{id}} \times \\
& \quad \times \sqrt{\frac{m_{\mathrm{i}}}{k \times T_{\mathrm{e}}}} \times \mathrm{e}^{n_{\mathrm{n}} \times \sigma \times L_{\mathrm{s}}} \times\left(1-\mathrm{e}^{-n_{\mathrm{n}} \times \sigma \times L_{\mathrm{f}}}\right) .
\end{aligned}
$$

Cette formule est une généralisation de celles établies par Clayden et Hurdle d'une part [4], et Sajben et Blumenthal d'autre part [13].

2.2 LA CHAMBRE DE PTS. - Nous allons maintenant évaluer numériquement cette expression en fonction des paramètres et des variables expérimentales correspondants à la chambre utilisée à PTS [5].

2.2.1 Paramètres expérimentaux. - On suppose qu'ils n'ont pas varié au cours des diverses expériences.
* Espèce chimique une fois ionisée : argon

$$
\begin{gathered}
m_{\mathrm{i}} \approx 36 \times 1,67 \times 10^{-27} \approx 6 \times 10^{-26} \mathrm{~kg} \\
\mathrm{Ar}_{\mathrm{d}}^{+}+\mathrm{Ar}_{1} \rightarrow \mathrm{Ar}_{\mathrm{d}}+\mathrm{Ar}_{1}^{+} \\
E\left(\mathrm{Ar}_{\mathrm{d}}^{+}\right)=20-60 \mathrm{eV} \Rightarrow \sigma \approx 4( \pm 1) \times 10^{-19} \mathrm{~m}^{2}
\end{gathered}
$$

* Température des atomes neutres et des ions lents :

L'échange de charge résonnant conduit à :

$$
T_{\mathrm{il}} \approx T_{\mathrm{n}} \approx 300 \mathrm{~K}=T .
$$

La température des parois externes de la chambre est toujours restée proche de cette valeur. Il existait cependant une paroi interne parfois refroidie à l'azote liquide.

* Température électronique mesurée par une sonde de Langmuir :

$$
T_{\mathrm{e}} \approx 1200 \pm 200 \mathrm{~K} .
$$

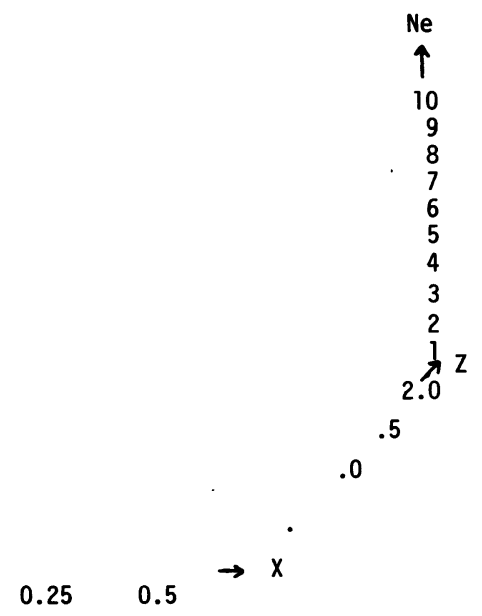

$\theta * 25^{\circ}$

Fig. 3. - Cartographie de la densité électronique ( $\mathrm{Ne}$ en $10^{5} \mathrm{~cm}^{-3}$ ) dans la chambre à plasma de PTS. $Z$ représente la coordonnée axiale, $X$ la coordonnée radiale. La source à plasma est située du côté des $Z>2$. Le bord du faisceau n'est pas net, d'où une grande incertitude sur la valeur de l'angle d'ouverture $\theta$.

[Density distribution (in $10^{5} \mathrm{~cm}^{-3}$ ) in the PTS plasma chamber. $Z$ represents the axial, and $X$ the radial coordinate (in meter). The plasma source is located at the largest densities $(Z>2)$. The aperture angle $\theta$ is not well defined because of the unsharpness of the beam edge.] 
* Dimensions de la chambre à plasma :

$$
R \approx 1,25 \mathrm{~m} \quad L \approx 5,50 \mathrm{~m} .
$$

* Angle d'ouverture du faisceau de plasma dérivant :

Ce paramètre est très mal connu car d'une part le faisceau n'est pas strictement homogène avec des bords bien nets et d'autre part une mesure de densité totale (électronique) ne le sépare pas du plasma froid, beaucoup plus diffus. Il n'a pas fait l'objet d'investigations systématiques et l'on peut douter de sa stabilité pendant toutes les expériences.

La figure 3 nous permet d'estimer très approximativement :

$$
\theta \approx 25^{\circ}\left( \pm 10^{\circ}\right)
$$

* Position de la sonde de mesure de densité :

Elle se trouvait placée entre la source de plasma et la maquette de mesure, dans le plasma supposé non perturbé :

$$
L_{\mathrm{s}} \approx 2 \mathrm{~m}
$$

* Longueur effective du faisceau de plasma dérivant :

C'est, rappelons-le, la longueur d'un faisceau uniquement cônique, équivalent au faisceau réel, vis-à-vis de la production d'ions lents. Cette longueur est évaluée numériquement par le calcul approché des intégrales concernées (5). Elle dépend de l'angle $\theta$ puisque l'on a par définition :

$$
\frac{R}{\operatorname{tg} \theta}<L_{\mathrm{f}}<L
$$

Numériquement, avec l'angle estimé plus haut $\left(\theta \approx 25^{\circ}\right)$, on trouve :

$$
L_{\mathrm{f}} \approx 4( \pm 1,5) \mathrm{m} \text {. }
$$

\subsubsection{Variables expérimentales.}

* Vitesse des ions dérivants :

Cette vitesse, mesurée par la tension d'accélération de la source à plasma (de type Kaufman) s'exprime donc à l'aide de l'énergie de dérive $U_{\mathrm{a}}$, en électron-Volt :

$$
\begin{aligned}
v_{\text {id }}\left[\mathrm{m} . \mathrm{s}^{-1}\right]=\sqrt{\frac{2 \times q}{m_{\mathrm{i}}}} \times \sqrt{U_{\mathrm{a}}} \approx 2,3 & \times 10^{3} \times \\
& \times \sqrt{U_{\mathrm{a}}}[\mathrm{eV}] .
\end{aligned}
$$

Compte tenu du mode de production du faisceau, la vitesse de dérive se superpose à une énergie thermique de l'ordre de quelques électrons-volt au maximum.

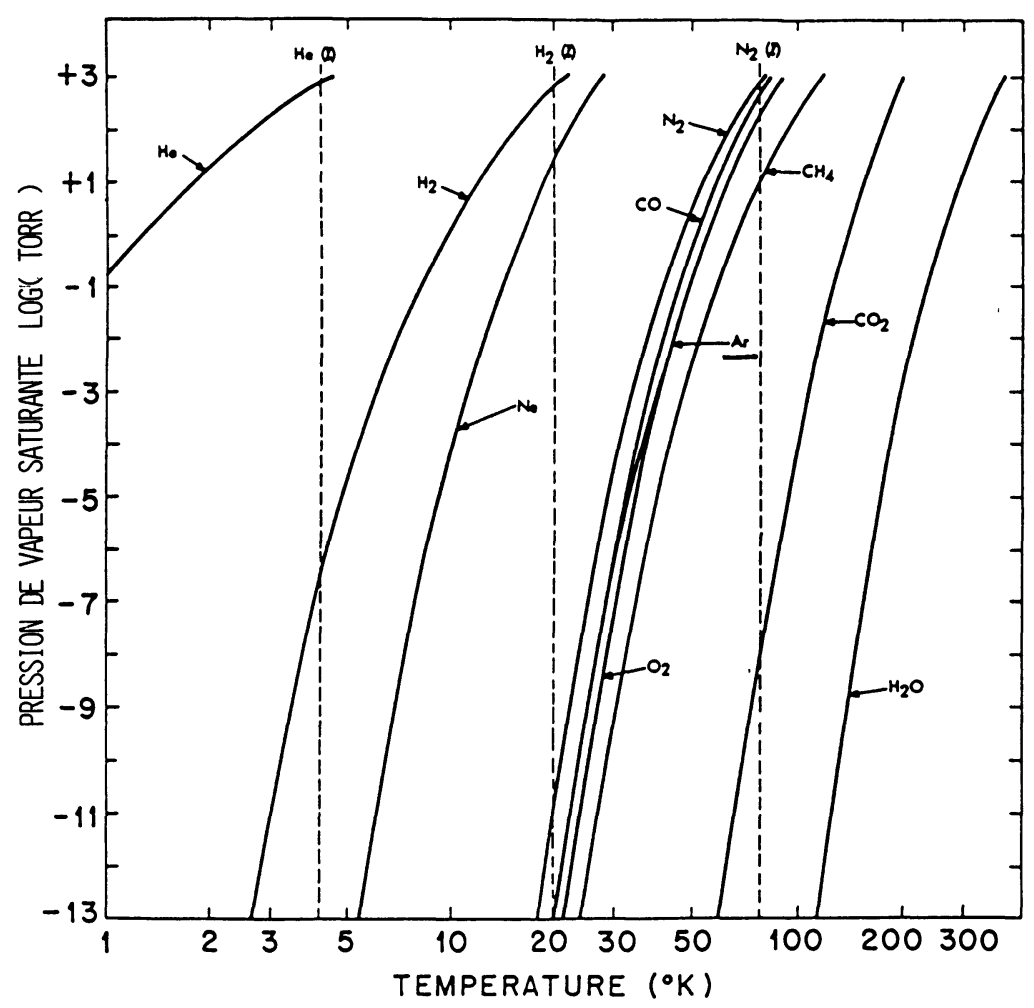

Fig. 4. - Pressions de vapeur saturante pour divers gaz courants dont l'argon. Les lignes verticales pointillées indiquent les températures d'ébullition de $\mathrm{N}_{2}, \mathrm{H}_{2}$ et $\mathrm{He}$ à 760 Torr $\left(\approx 10^{5} \mathrm{~N} \cdot \mathrm{m}^{-2}\right)$.

[Vapor pressures of argon and other common gases at low temperatures. Dashed lines indicate the boiling temperatures of $\mathrm{N}_{2}, \mathrm{H}_{2}$ and $\mathrm{He}$ at 760 Torr $\left(\approx 10^{5} \mathrm{~N} \cdot \mathrm{m}^{-2}\right)$.] 
* Densité des atomes neutres d'argon :

Le vide était obtenu à l'aide de deux pompes turbomoléculaires en série. Seules la pression totale $P_{\mathrm{n}}$ et parfois la pression en l'absence de plasma $P_{\mathrm{v}}$, ont été mesurées. En l'absence du plasma d'argon, on s'attend à ce que la pression résiduelle provienne essentiellement de molécules $\mathrm{H}_{2} \mathrm{O}$, ainsi qu'en plus faibles proportions, de $\mathrm{N}_{2}, \mathrm{O}_{2}, \mathrm{CO}_{2}$, $\mathrm{He}$... Cette hypothèse semble confirmée par la forte baisse de la pression $P_{\mathrm{v}}$ (facteur 10) observée lors de l'utilisation d'un écran cryogénique à azote liquide, qui piège surtout $\mathrm{H}_{2} \mathrm{O}$ et $\mathrm{CO}_{2}$, mais pas du tout l'argon (Fig. 4) :

- en l'absence d'écran :

$$
P_{\mathrm{v}} \approx 5 \times 10^{-6} \text { Torr } \approx 7 \times 10^{-4} \mathrm{~N} \cdot \mathrm{m}^{-2}
$$

- en présence de l'écran:

$$
\begin{gathered}
P_{\mathrm{v}} \approx 6 \times 10^{-7} \text { Torr } \approx 8 \times 10^{-5} \text { N.m }{ }^{-2} \\
\left(1 \text { Torr } \approx 133 \text { N.m }{ }^{-2}\right) .
\end{gathered}
$$

Nous supposerons alors que la pression partielle d'argon en présence du plasma est approximativement :

$$
P_{\mathrm{Ar}} \approx P_{\mathrm{n}}-P_{\mathrm{v}} .
$$

La densité d'atomes neutres notée $n_{\mathrm{n}}$ (paragraphe 2.1), ne représente que les atomes d'argon. On la relie aux pressions mesurées par:

$$
n_{\mathrm{n}}=n_{\mathrm{Ar}}=\frac{P_{\mathrm{Ar}}}{k \times T} \approx \frac{P_{\mathrm{n}}-P_{\mathrm{v}}}{k \times T} .
$$

Avec $T \approx 300 \mathrm{~K}$ et les pressions en Torr, on $\mathrm{a}:$

$$
n_{\mathrm{Ar}}\left[\mathrm{m}^{-3}\right] \approx 3,2 \times 10^{22} \times\left(P_{\mathrm{n}}-P_{\mathrm{v}}[\text { Torr }]\right) .
$$

* La densité du plasma, à la distance $L_{\mathrm{s}}$ de la source à plasma était mesurée par une sonde à impédance mutuelle dont la résonance indique la fréquence plasma électronique. Celle-ci est reliée de façon simple à la densité électronique :

$$
f_{\mathrm{p}}=\frac{\omega_{\mathrm{p}}}{2 \times \pi}=\frac{1}{2 \times \pi} \times \sqrt{\frac{n_{\mathrm{e}} \times q^{2}}{\varepsilon_{0} \times m_{\mathrm{e}}}} .
$$

La neutralité macroscopique du plasma dans la zone de mesure, non perturbée, permet d'écrire numériquement :

$$
n_{\mathrm{il}}+n_{\mathrm{id}}=n_{\mathrm{e}}\left[\mathrm{m}^{-3}\right] \approx 1,2 \times 10^{10} \times\left(f_{\mathrm{p}}[\mathrm{MHz}]\right)^{2} .
$$

Cette fréquence plasma, comprise entre 2 et 10 $\mathrm{MHz}$ est connue à $0,1 \mathrm{MHz}$ près, d'où une incertitude sur la densité ionique comprise entre 2 et $10 \%$.

2.2.3 Expression de $n_{\mathrm{il}}$ en fonction des variables expérimentales. - En remplaçant dans l'expression (8), les différentes variables et paramètres, on obtient :

$$
\begin{aligned}
\frac{n_{\mathrm{il}}}{n_{\mathrm{id}}}=C_{1} \times \sqrt{U_{\mathrm{a}}} \times \mathrm{e}^{C_{2} \times\left(P_{\mathrm{n}}-P_{\mathrm{v}}\right)} \times & \\
& \times\left(1-\mathrm{e}^{-C_{3} \times\left(P_{\mathrm{n}}-P_{\mathrm{v}}\right)}\right) .
\end{aligned}
$$

Avec :

$$
\begin{aligned}
& C_{1} \approx 0,82 \times \frac{\left(L_{\mathrm{s}} \times \operatorname{tg} \theta\right)^{2}}{R \times(R+L)} \times \sqrt{\frac{2 \times q}{k \times T_{\mathrm{e}}}} \\
& C_{2} \approx \frac{\sigma \times L_{\mathrm{s}}}{k \times T} \\
& C_{3} \approx \frac{\sigma \times L_{\mathrm{f}}}{k \times T} .
\end{aligned}
$$

Numériquement, compte tenu des incertitudes sur les paramètres, nous avons estimé les fourchettes suivantes pour les valeurs de ces constantes :

$$
\begin{aligned}
0,05 & <C_{1}<1,5 \\
10^{4} & <C_{2}<5 \times 10^{4} \\
3 \times 10^{4} & <C_{3}<7 \times 10^{4} .
\end{aligned}
$$

La connaissance du rapport et de la somme des densités ioniques nous permet d'exprimer la densité d'ions lents :
2.2.4 Courant d'ions lents. - Afin d'exprimer le courant d'ions lents collecté par la plaque carrée, polarisée négativement et située à la surface de la maquette cylindrique, elle-même à la masse (Fig. 1), nous faisons l'hypothèse que la densité moyenne d'ions lents n'est pas perturbée par la collection sur cette surface. Dans ce cas, on peut écrire la proportionnalité :

$$
I_{\mathrm{il}} \propto n_{\mathrm{il}} .
$$

Cette hypothèse est certainement justifiée par une extension faible de la perturbation de potentiel, 
écrantée rapidement par le plasma environnant $\left(\lambda_{\mathrm{D}} \leqslant 1 \mathrm{~cm}\right)$.

Par ailleurs, l'observation des résultats expérimentaux (Fig. 5) conduit à poser aussi la proportionnalité suivante :

$$
I_{\mathrm{il}} \propto \varphi
$$

où $\varphi$ est le potentiel appliqué à la plaque collectrice. Bien entendu, lorsque $\varphi$ est nul (plaque à la masse), le courant ne doit pas être strictement nul. Ceci n'arrive en effet que pour le potentiel flottant. Mais ce courant de diffusion simple, provenant d'une agitation thermique d'énergie inférieure à $1 \mathrm{eV}$, est certainement faible devant les courants mesurés pour des polarisations négatives supérieures à une dizaine de volts.

Ainsi, nous sommes amenés à écrire la relation suivante :

$$
I_{\mathrm{il}}=C_{4} \times n_{\mathrm{il}} \times \varphi
$$

où le coefficient de proportionnalité $C_{4}$, ne dépendant que de la géométrie de collection, doit être établi empiriquement.

Cette relation est à rapprocher du résultat de Laframboise [8], concernant le courant ionique

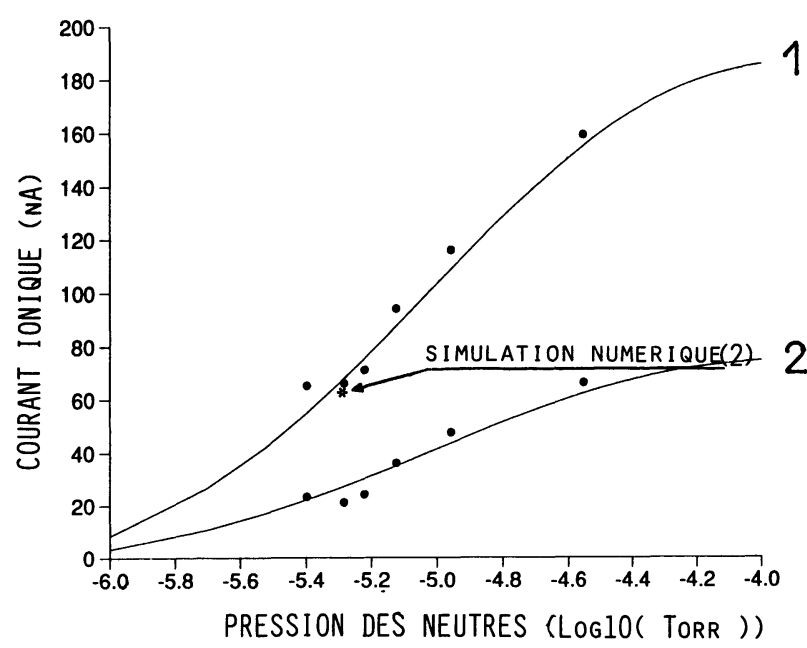

Fig. 5. - Comparaison entre les mesures $(\bullet)$ et le modèle d'interprétation (-). Variation du courant collecté avec la pression totale. Les paramètres sont: $F_{\mathrm{p}} \approx 5 \mathrm{MHz}$; $U_{\mathrm{a}} \approx 60(\mathrm{eV}) ; \quad P_{\mathrm{v}} \approx 6 \times 10^{-7}$ Torr $\approx 8 \times 10^{-5} \mathrm{~N} \cdot \mathrm{m}^{-2}$. Avec pour chacune des courbes: 1) polarisation: $\varphi \approx-50$ Volt, 2) polarisation : $\varphi \approx-20$ Volt. On a aussi indiqué le résultat obtenu par simulation numérique (paragraphe 3) pour le cas 2).

[Comparison between experimental data $(\bullet)$ and the results of our model (-). Ionic current versus pressure with following parameters: $F_{\mathrm{p}} \approx 5 \mathrm{MHz} ; U_{\mathrm{a}} \approx 60(\mathrm{eV})$; $P_{\mathrm{v}} \approx 6 \times 10^{-7}$ Torr $\approx 8 \times 10^{-5} \mathrm{~N} . \mathrm{m}^{-2}$. For each curve $\left.: 1\right)$ polarisation: $\varphi \approx-50$ Volt, 2) polarisation: $\varphi \approx$ -20 Volt. Also shown is the result of a numerical simulation (Sect. 3) with $\varphi \approx-20$ Volt.] collecté par une sonde sphérique polarisée négativement, dans un plasma non collisionnel :

$$
\begin{aligned}
& I=n_{\infty} \times \\
& \times\left[\sqrt{\frac{k \times T_{\mathrm{i}}}{2 \times \pi \times m_{\mathrm{i}}}}-\frac{q \times \varphi}{\sqrt{2 \times \pi \times m_{\mathrm{i}} \times k \times T_{\mathrm{i}}}}\right] \times \\
& \times \begin{array}{l}
q \times S_{\text {collection }} \\
{[}
\end{array}
\end{aligned}
$$

où l'on négligerait le premier des deux termes entre crochets.

Dans notre cas cependant, la géométrie du système expérimental conduit plutôt une collection hémisphérique.

2.2.5 Résultats. - Les quatre coefficients définis plus haut (relations (9) et (11) ont été ajustés, dans des limites réalistes pour les trois premiers, afin de rendre compte au mieux de l'ensemble des résultats de mesures (Figs. 5 à 8 ).

Avec les unités suivantes pour les variables :

$$
I[\mathrm{nA}], \varphi[\text { Volt }], U_{\mathrm{a}}[\mathrm{eV}], P_{\mathrm{n}}[\mathrm{Torr}], f_{\mathrm{p}}[\mathrm{MHz}]
$$

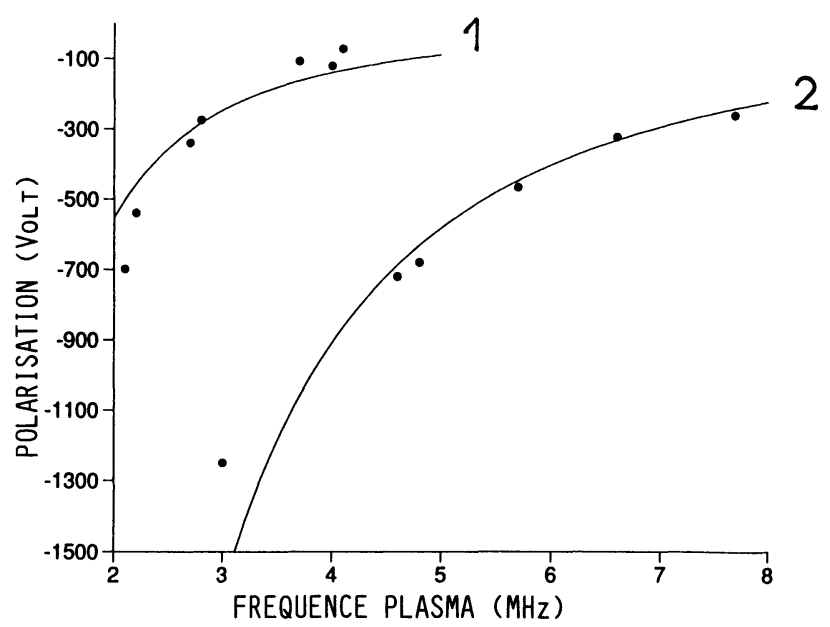

Fig. 6. - Comparaison entre les mesures (•) et le modèle d'interprétation (-). Variation de la polarisation (surface alimentée par un courant électronique constant) avec la fréquence plasma (densité). Les paramètres sont: $U_{\mathrm{a}} \approx$ $60(\mathrm{eV}) ; \quad P_{\mathrm{v}} \approx 5 \times 10^{-6}$ Torr $\approx 6,6 \times 10^{-4} \mathrm{~N} \cdot \mathrm{m}^{-2}$. Avec pour chacune des courbes: 1) courant électronique : $I \approx 35 \mathrm{nA} ; P_{\mathrm{n}} \approx 6 \times 10^{-6}$ Torr $\approx 8 \times 10^{-4} \mathrm{~N} \cdot \mathrm{m}^{-2}, 2$ ) courant électronique : $I \approx 30 \mathrm{nA} ; \quad P_{\mathrm{n}} \approx 5,12 \times$ $10^{-6}$ Torr $\approx 6,8 \times 10^{-4}$ N.m ${ }^{-2}$.

[Comparison between experimental data $(\bullet)$ and the results of our model (-). Polarisation (rear surface supplied with a constant electronic current) versus plasma frequency (density), with following parameters: $U_{\mathrm{a}} \approx$ $60(\mathrm{eV}) ; \quad P_{\mathrm{v}} \approx 5 \times 10^{-6}$ Torr $\approx 6,6 \times 10^{-4} \mathrm{~N} \cdot \mathrm{m}^{-2}$. For each curve : 1) electronic current: $I \approx 35 \mathrm{nA} ; P_{\mathrm{n}} \approx$ $6 \times 10^{-6}$ Torr $\left.\approx 8 \times 10^{-4} \mathrm{~N} . \mathrm{m}^{-2}, 2\right)$ electronic current : $I \approx 30 \mathrm{nA} ; P_{\mathrm{n}} \approx 5.12 \times 10^{-6}$ Torr $\approx 6.8 \times 10^{-4} \mathrm{~N} \cdot \mathrm{m}^{-2}$.] 


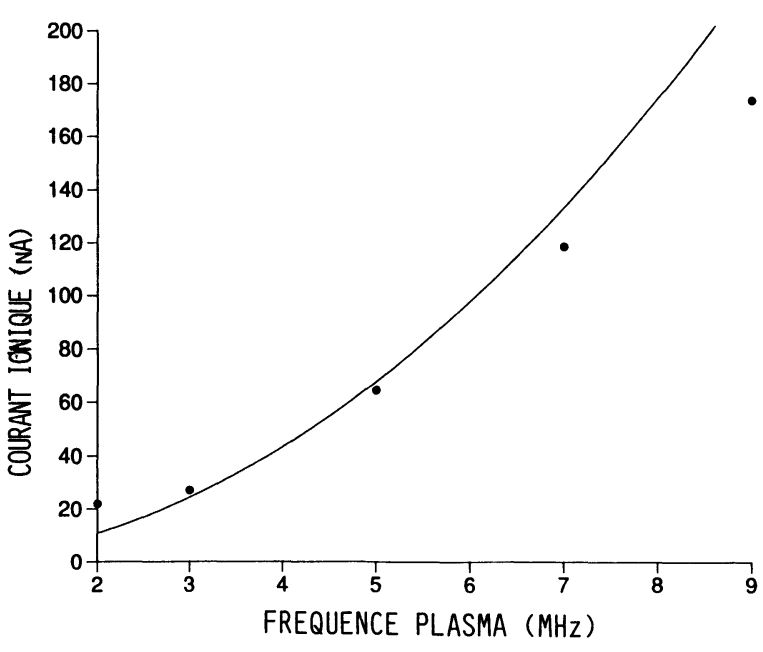

Fig. 7. - Comparaison entre les mesures $(\bullet)$ et le modèle d'interprétation (-). Variation du courant collecté avec la fréquence plasma (densité). Les paramètres sont: $\varphi \approx-50$ Volt $; P_{\mathrm{n}} \approx 5,2 \times 10^{-6}$ Torr $\approx 6,9 \times 10^{-4} \mathrm{~N} . \mathrm{m}^{-2} ;$ $P_{\mathrm{v}} \approx 6 \times 10^{-7}$ Torr $\approx 8 \times 10^{-5}$ N.m ${ }^{-2} ; U_{\mathrm{a}} \approx 60 \mathrm{eV}$.

[Comparison between experimental data $(\bullet)$ and the results of our model (-). Ionic current versus plasma frequency (density), with following parameters : $\varphi \approx-50$ Volt $; P_{\mathrm{n}} \approx 5.2 \times 10^{-6}$ Torr $\approx 6.9 \times 10^{-4} \mathrm{~N} \cdot \mathrm{m}^{-2} ;$ $P_{\mathrm{v}} \approx 6 \times 10^{-7}$ Torr $\approx 8 \times 10^{-5}$ N.m ${ }^{-2} ; U_{\mathrm{a}} \approx 60 \mathrm{eV}$.]

on trouve numériquement :

$$
\begin{aligned}
& C_{1} \approx 0,3 \\
& C_{2} \approx 3,7 \times 10^{4} \\
& C_{3} \approx 5 \times 10^{4} \\
& C_{4} \approx 1,2 \times 10^{-5} .
\end{aligned}
$$

Les trois premiers permettent de « remonter » à des valeurs plausibles de certains paramètres mal connus :

$L_{\mathrm{s}} \approx \frac{C_{2} \times k \times T}{\sigma} \approx 2,5 \mathrm{~m}$

$\theta \approx \operatorname{arctg} \sqrt{C_{1} \times 1,2 \times \frac{R \times(R+L)}{L_{\mathrm{s}}^{2}} \times \sqrt{\frac{k \times T_{\mathrm{e}}}{2 \times q}} \approx 18^{\circ}}$

$L \approx \frac{C_{3} \times k \times T}{\sigma} \approx 3 \mathrm{~m}$.

On constate en fait une incompatibilité entre la valeur de l'angle $\theta$ et la longueur. $L_{\mathrm{f}}$, en effet :

$$
\frac{R}{\operatorname{tg} \theta} \approx 3,8 \mathrm{~m}>L_{\mathrm{f}} \text {. }
$$

Il ne faut cependant pas oublier toutes les approximations et simplifications faites dans ce modèle. Outre la connaissance imparfaite de certains paramètres $\left(\sigma, T_{\mathrm{e}}\right.$ ?), l'hypothèse d'un faisceau de plasma cônique homogène est sûrement trop schématique.

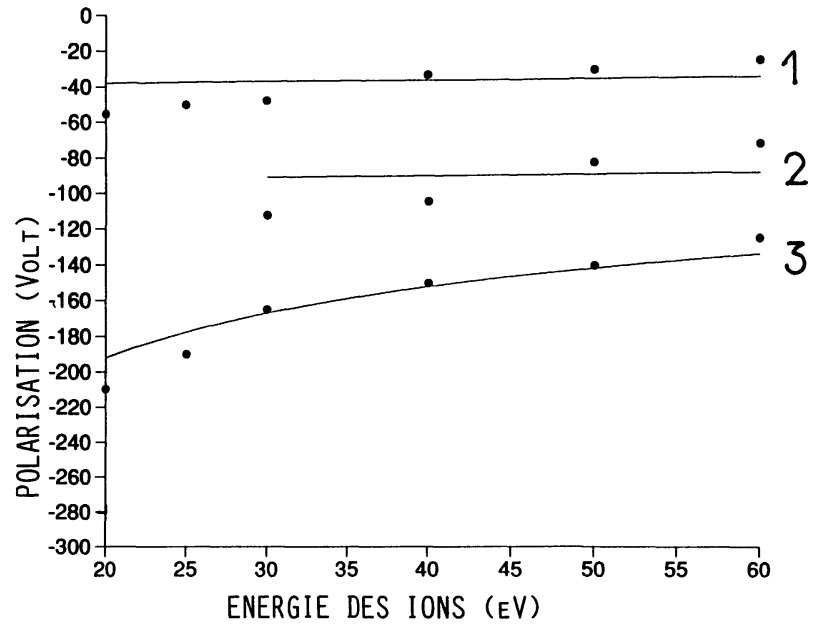

Fig. 8. - Comparaison entre les mesures $(\bullet)$ et le modèle d'interprétation (-). Variations de la polarisation (surface alimentée par un courant électronique constant) avec l'énergie des ions dérivants. Les paramètres sont : $P_{\mathrm{v}} \approx$ $5 \times 10^{-6}$ Torr $\approx 6,6 \times 10^{-4} \mathrm{~N} \cdot \mathrm{m}^{-2}$. Avec pour chacune des courbes : 1) $I \approx 100 \mathrm{nA} ; \quad P_{\mathrm{n}} \approx 3,5 \times 10^{-5}$ Torr $\approx$ $\left.4,6 \times 10^{-3} \mathrm{~N} \cdot \mathrm{m}^{-2} ; F_{\mathrm{p}} \approx 4,8 \mathrm{MHz}, 2\right) I \approx 65 \mathrm{nA} ; P_{\mathrm{n}} \approx$ $5,5 \times 10^{-5}$ Torr $\approx 7,3 \times 10^{-3}$ N.m $\left.{ }^{-2} ; \quad F_{\mathrm{p}} \approx 2,3 \mathrm{MHz}, 3\right)$ $I \approx 100 \mathrm{nA} ; P_{\mathrm{n}} \approx 1,05 \times 10^{-5}$ Torr $\approx 1,4 \times 10^{-3} \mathrm{~N} \cdot \mathrm{m}^{-2} ;$ $F_{\mathrm{p}} \approx 3,5 \mathrm{MHz}$.

[Comparison between experimental data (•) and the results of our model (-). Polarisation (rear surface supplied with a constant electronic current) versus ions drift energy, with following parameters: $P_{\mathrm{v}} \approx 5 \times$ $10^{-6}$ Torr $\approx 6.6 \times 10^{-4} \mathrm{~N} . \mathrm{m}^{-2}$. For each curve: 1) $I \approx 100 \mathrm{nA} ; \quad P_{\mathrm{n}} \approx 3.5 \times 10^{-5}$ Torr $\approx 4.6 \times 10^{-3} \mathrm{~N} \cdot \mathrm{m}^{-2} ;$ $\left.F_{\mathrm{p}} \approx 4.8 \mathrm{MHz}, 2\right) \quad I \approx 65 \mathrm{nA} ; \quad P_{\mathrm{n}} \approx 5.5 \times 10^{-5}$ Torr $\approx$ $\left.7.3 \times 10^{-3} \mathrm{~N} . \mathrm{m}^{-2} ; F_{\mathrm{p}} \approx 2.3 \mathrm{MHz}, 3\right) I \approx 100 \mathrm{nA} ; P_{\mathrm{n}} \approx$ $1.05 \times 10^{-5}$ Torr $\approx 1.4 \times 10^{-3}$ N.m ${ }^{-2} ; F_{\mathrm{p}} \approx 3.5 \mathrm{MHz}$.]

Par ailleurs, une série de mesures concernant la variation du courant collecté avec l'énergie de dérive, reste inexpliquée (Fig. 9) et contradictoire avec les précédentes. Ce résultat pourrait s'interpréter dans ce cas précis, par une stabilisation insuffisante du plasma entre des mesures trop rapprochées, le faisceau se rétrécissant lorsque la vitesse de dérive augmente (Pigache, communication privée). Suppo-

$$
\operatorname{tg} \theta \approx \frac{v_{\perp}}{v_{\|}} \approx \frac{\text { Cste }}{\sqrt{U_{\mathrm{a}}}} .
$$

Le sens de variation devient alors plus conforme à l'observation (Fig. 10). On peut aussi envisager une légère dérive de la fréquence plasma (i.e. en fait une variation non adéquate du courant émis par la source de plasma), à laquelle le courant d'ions lents est très sensible, et qui était parfois insuffisamment précise (paragraphe 2.2.2). 


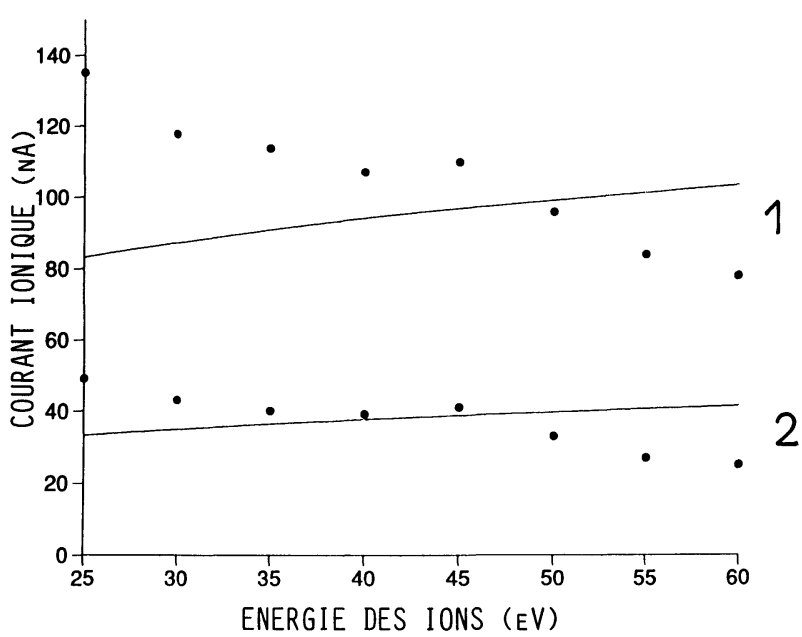

Fig. 9. - Comparaison entre les mesures $(\bullet)$ et le modèle d'interprétation (-). Variation du courant collecté avec l'énergie des ions dérivants. Les paramètres constants sont : $\quad F_{\mathrm{p}} \approx 5 \mathrm{MHz} ; \quad P_{\mathrm{v}} \approx 6 \times 10^{-7}$ Torr $\approx 8 \times$ $10^{-5} \mathrm{~N} . \mathrm{m}^{-2} ; P_{\mathrm{n}} \approx 10^{-5}$ Torr $\approx 1,3 \times 10^{-3} \mathrm{~N} . \mathrm{m}^{-2}$. Avec pour chacune des courbes: 1) polarisation: $\varphi \approx-50$ Volt, 2) polarisation : $\varphi \approx-20$ Volt. On constate l'accord sur la valeur moyenne, mais le désaccord quant au sens des variations. Le modèle "brut " ne rend pas compte de cette série d'expériences.

[Comparison between experimental data $(\bullet)$ and the results of our model (-). Ionic current versus ions drift energy, with following parameters: $F_{\mathrm{p}} \approx 5 \mathrm{MHz}$; $P_{\mathrm{v}} \approx 6 \times 10^{-7}$ Torr $\approx 8 \times 10^{-5}$ N.m ${ }^{-2} ; P_{\mathrm{n}} \approx 10^{-5}$ Torr $\approx$ $1.3 \times 10^{-3} \mathrm{~N} . \mathrm{m}^{-2}$. For each curve: 1) polarisation : $\varphi \approx-50$ Volt, 2) polarisation : $\varphi \approx-20$ Volt. The initial model does not fit correctly the experimental data, although the mean value is in the good range.]

2.3 CHAMBRE DE L'ONERA. - Nous avons par ailleurs, appliqué cette formulation analytique au cas de mesures réalisées par Fournier et Pigache dans les conditions particulièrement bien définies de l'ancienne chambre à plasma de l'ONERA (Fig. 11) [7].

\subsubsection{Paramètres expérimentaux.}

* Le gaz ionisé était encore de l'argon et la température des neutres était toujours proche de $300 \mathrm{~K}$, même en présence d'une paroi refroidie cette fois-ci à l'hélium liquide.

* La température électronique était de l'ordre de $: T_{\mathrm{e}} \approx 1500 \mathrm{~K}$.

* Les dimensions de la chambre sont analogues à celle de PTS :

$$
R \approx 1,5 \mathrm{~m} \quad L \approx 5 \mathrm{~m}
$$

* Le demi-angle d'ouverture du faisceau d'ions dérivant est ici très bien déterminé, grâce à l'existence d'un tube collimateur (Fig. 11):

$$
\theta \approx 10^{\circ} \text {. }
$$

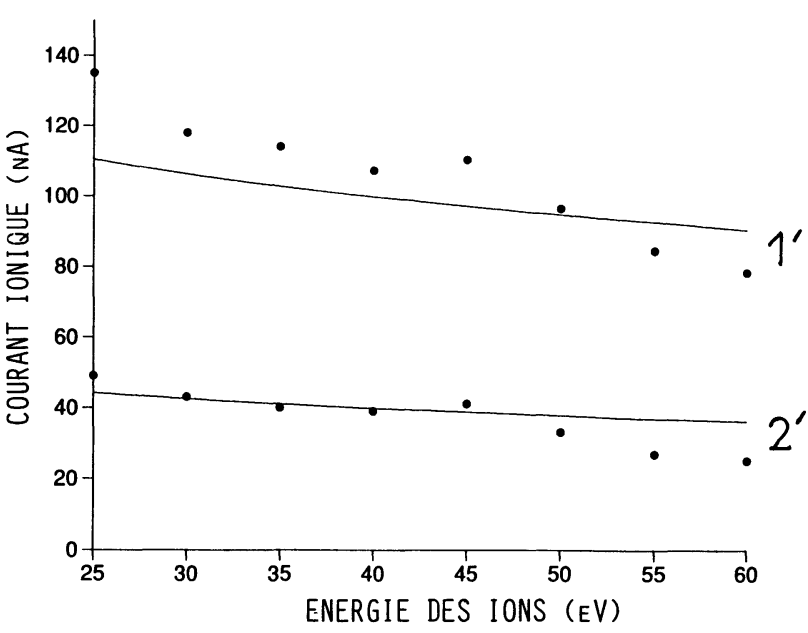

Fig. 10. - Les paramètres et les mesures sont les mêmes que pour la figure 9 . Seul le modèle a changé : on suppose maintenant une variation de l'angle d'ouverture $\theta$ avec l'énergie des ions dérivants $\left(\operatorname{tg}(\theta) \propto\left(U_{\mathrm{a}}\right)^{-1 / 2}\right)$. On voit que l'accord est nettement meilleur.

[Data and parameters are identical to those of figure 9. Only the model is changed : we assume a variation of the beam aperture angle with drift energy $\left(\operatorname{tg}(\theta) \propto\left(U_{\mathrm{a}}\right)^{-1 / 2}\right)$ what enables a better fit to be achieved.]

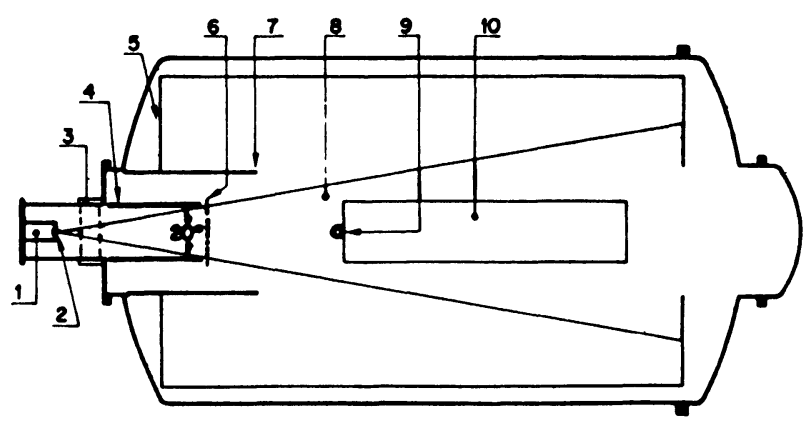

Fig. 11. - Schéma de la chambre à plasma de l'ONERA. 1) source d'ions, 2) sortie de la source d'ion, 3) valve, 4) tube collimateur refroidi à l'hélium liquide, 5) écran métallique interne, 6) grille (pour simuler une température transversale des ions dérivants), 7) écran en aluminium poli, 8) faisceau de plasma, 9) obstacle sphérique, 10) région explorée par une sonde de Langmuir.

[Schematic of the experimental arrangement of ONERA. 1) ion source, 2) ion source opening, 3) valve, 4) liquidhelium trap, 5) stainless steel screen, 6) grid (for simulation of temperature), 7) polished aluminium screen, 8) ion beam, 9) sphere, 10) zone explored with a Langmuir probe.]

* La longueur effective du faisceau est ici celle de la chambre, car l'angle d'ouverture est trop petit pour que les ions dérivants atteignent les parois latérales :

$$
L_{\mathrm{f}}=L \approx 5 \mathrm{~m}
$$


* Position de la sonde de mesure :

$$
L_{\mathrm{s}} \approx 3 \mathrm{~m}
$$

Ces valeurs nous permettent de calculer les coefficients définis plus haut:

$$
\begin{aligned}
& C_{1} \approx 0,093\left[\mathrm{eV}^{-0,5}\right] \\
& C_{2} \approx 3,9 \times 10^{4}\left[\text { Torr }^{-1}\right] \approx 290\left[\left(\mathrm{~N} \cdot \mathrm{m}^{-2}\right)^{-1}\right] \\
& C_{3} \approx 6,4 \times 10^{4}\left[\text { Torr }^{-1}\right] \approx 480\left[\left(\mathrm{~N} \cdot \mathrm{m}^{-2}\right)^{-1}\right] .
\end{aligned}
$$

2.3.2 Variables expérimentales. - Fournier et Pigache ne présentent qu'une seule série de mesures où les ions lents sont importants (Fig. 3 de leur article), pour laquelle seule la pression des neutres diffère de celle mesurée dans une autre série de mesures (Fig. 4 de leur article). Ces résultats sont réunies dans la figure 12 .

* Dans les deux cas la vitesse de dérive était analogue, correspondant à une énergie :

$$
U_{\mathrm{a}} \approx 20 \mathrm{eV} .
$$

* La pression était bien contrôlée grâce à une paroi refroidie à l'hélium liquide, qui piégeait l'argon (Fig. 4). Nous ferons alors l'hypothèse que la pression résiduelle provenait essentiellement d'atomes d'argon émis par la source à plasma et non piégés.

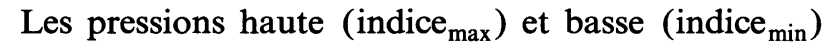
furent respectivement :

$$
\begin{aligned}
& P_{\text {max }} \approx 2 \times 10^{-4} \text { N.m } \mathrm{m}^{-2} \approx 1,5 \times 10^{-6} \text { Torr } \\
& P_{\text {min }} \approx 1,5 \times 10^{-5} \text { N.m }{ }^{-2} \approx 1,13 \times 10^{-7} \text { Torr } .
\end{aligned}
$$

On en déduit, d'après l'expression (9) et compte tenu des coefficients calculés plus haut:

$$
\begin{aligned}
& \left(\frac{n_{\mathrm{il}}}{n_{\mathrm{id}}}\right)_{\max } \approx 0,04 \\
& \left(\frac{n_{\mathrm{il}}}{n_{\mathrm{id}}}\right)_{\text {min }} \approx 0,003 .
\end{aligned}
$$

* La densité ionique totale hors du sillage, s'identifie à la densité électronique, que l'on peut déterminer à l'aide de la longueur de Debye $\lambda_{\mathrm{D}}$ et de la empera ure e ec ronique e ig. . e es pra 1quement la même pour les deux séries de mesures :

$$
n_{\mathrm{e}} \approx n_{\mathrm{id}}+n_{\mathrm{il}} \approx \frac{\varepsilon_{0} \times k \times T_{\mathrm{e}}}{\left(q \times \lambda_{\mathrm{D}}\right)^{2}} \approx 1,5 \times 10^{12} \mathrm{~m}^{-3}
$$

On peut alors déterminer les densités des ions dérivants et lents pour chacune des deux séries de mesures :

$$
\begin{aligned}
& \left(n_{\mathrm{i} 1}\right)_{\max } \approx 5,8 \times 10^{10} \mathrm{~m}^{-3} \Rightarrow n_{\mathrm{id}} \approx 1,4 \times 10^{12} \mathrm{~m}^{-3} \\
& \left(n_{\mathrm{i} 1}\right)_{\min } \approx 4,5 \times 10^{9} \mathrm{~m}^{-3} \Rightarrow n_{\mathrm{id}} \approx 1,5 \times 10^{12} \mathrm{~m}^{-3}
\end{aligned}
$$

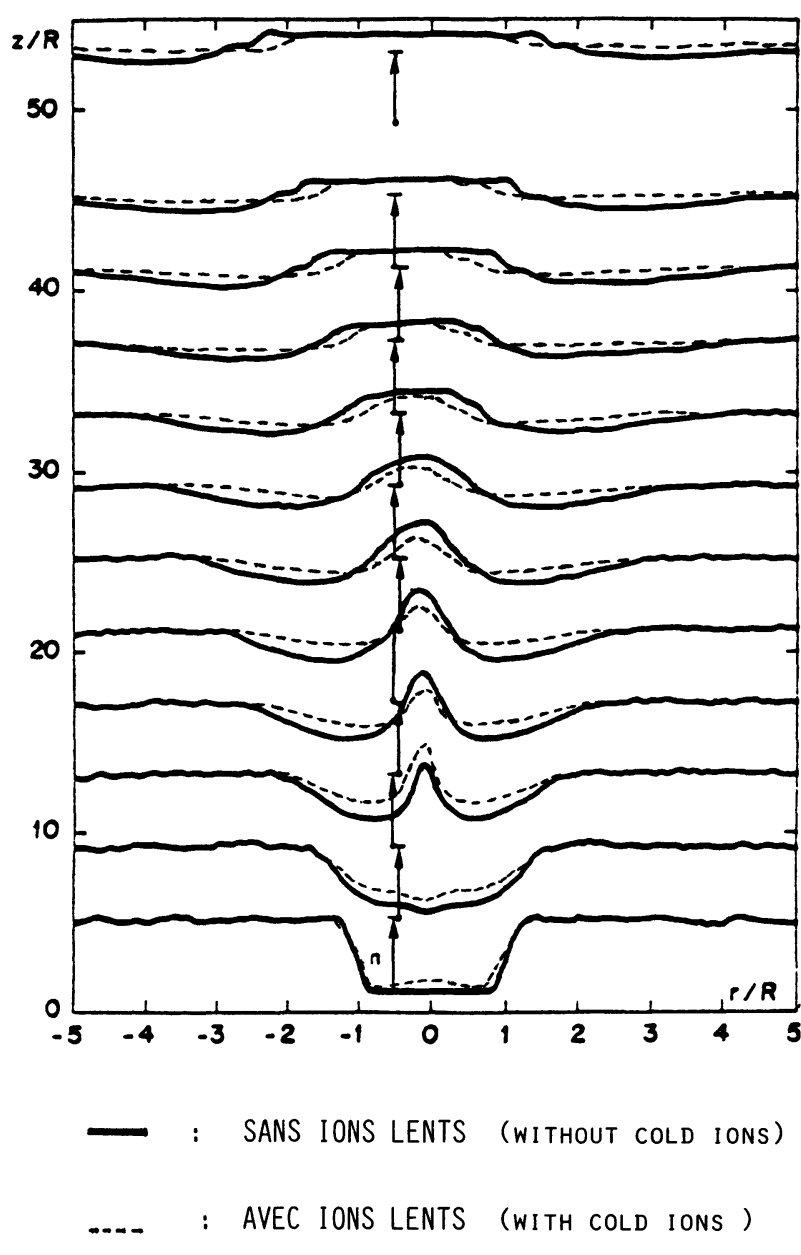

Fig. 12 - Mesures du courant ionique (d'après [7]) dans le sillage d'une sphère de $100 \mathrm{~mm}$ de diamètre, avec les paramètres suivants pour le plasma: $h$ (longueur de Debye) $\approx 2 \mathrm{~mm} ; T_{\mathrm{e}} \approx 1500 \mathrm{~K} ; U_{\mathrm{a}} \approx 20 \mathrm{eV} ; T_{\mathrm{e}} / T_{\mathrm{i}} \approx 10$. En trait plein, la pression basse: $P_{\mathrm{n}} \approx 1,13 \times$ $10^{-7}$ Torr $\approx 1,5 \times 10^{-5} \mathrm{~N} \cdot \mathrm{m}^{-2}$. En pointillés, la pression élevée : $P_{\mathrm{n}} \approx 1,5 \times 10^{-6}$ Torr $\approx 2 \times 10^{-4}$ N.m ${ }^{-2}$.

[Ionic currents (after [7]) in the wake of a floating sphere $100 \mathrm{~mm}$ diam. with following plasma parameters : $h$ (Debye Length) $\approx 2 \mathrm{~mm} ; \quad T_{\mathrm{e}} \approx 1500 \mathrm{~K} ; \quad U_{\mathrm{a}} \approx 20 \mathrm{eV}$; $T_{\mathrm{e}} / T_{\mathrm{i}} \approx 10$. Full lines: lower pressure: $P_{\mathrm{n}} \approx 1.13 \times$ $10^{-7}$ Torr $\approx 1.5 \times 10^{-5} \mathrm{~N} . \mathrm{m}^{-2}$. Broken line : higher pressure : $P_{\mathrm{n}} \approx 1.5 \times 10^{-6}$ Torr $\approx 2 \times 10^{-4}$ N.m ${ }^{-2}$.]

En pratique, les courants ioniques ont été mesurés par une sonde sphérique de 5 millimètres de diamètre, portée au potentiel $\varphi_{\text {sonde }}=-0,65$ Volt par rapport à la masse et normalisés par rapport au courant total hors du sillage.

* Les ions dérivants ont une énergie mono-directionnelle grande $(20 \mathrm{eV})$ devant la polarisation de la sonde, ils sont donc collectés essentiellement par la section de la sonde, indépendamment du potentiel de cette dernière :

$$
\begin{aligned}
i_{\mathrm{id}} \approx n_{\mathrm{id}} \times v_{\mathrm{id}} \times \pi \times r^{2} & \times q \approx \\
& \approx n_{\mathrm{id}} \times 3,2 \times 10^{-20} \mathrm{~A} .
\end{aligned}
$$


* Les ions lents à l'inverse, ont une énergie faible $(0,03 \mathrm{eV})$ et leur distribution de vitesse est supposée isotrope. Dans ce cas, nous pouvons appliquer la formule (12) établie par Laframboise [8] :

$$
\begin{aligned}
i_{\mathrm{il}} \approx n_{\mathrm{il}} & \times \sqrt{\frac{k \times T_{\mathrm{il}}}{2 \times \pi \times m_{\mathrm{i}}}} \times \\
& \times\left(1-\frac{q \times \phi}{k \times T_{\mathrm{il}}}\right) \times 4 \times \pi \times r^{2} \times q .
\end{aligned}
$$

Le potentiel $\phi$ est mesuré par rapport au potentiel plasma, il nous faut donc calculer le potentiel de la paroi (i.e. la masse) par rapport à ce dernier. Nous allons passer par le potentiel flottant de la sphère principale. Fournier et Pigache indique pour celle-ci un potentiel flottant par rapport à la paroi de l'ordre de :

$$
\varphi_{\text {flottant }} \approx \frac{q \times(-3)}{k \times T_{\mathrm{e}}} \approx-0,39 \text { Volt }
$$

Par ailleurs, on peut calculer ce potentiel flottant par rapport au plasma en écrivant la nullité de la somme des courants collectés :

$$
i_{\text {id }}+i_{\text {il }}+i_{\mathrm{e}}=0
$$

avec :

$$
\begin{aligned}
i_{\mathrm{il}} \ll & i_{\mathrm{id}} \\
i_{\mathrm{e}} \approx & n_{\mathrm{e}} \times \sqrt{\frac{2 \times k \times T_{\mathrm{e}}}{m_{\mathrm{e}}}} \times 2 \times \sqrt{\pi} \times R^{2} \times q \times \\
& \times \mathrm{e}^{\frac{\mathrm{q} \times \phi_{\text {flottant }}}{k \times T_{\mathrm{e}}}} \\
n_{\mathrm{e}}= & n_{\mathrm{il}}+n_{\mathrm{id}} \approx n_{i d}
\end{aligned}
$$

et en utilisant la relation (14), on déduit :

$$
\begin{aligned}
& \phi_{\text {flottant }} \approx \frac{k \times T_{\mathrm{e}}}{2 \times q} \times \operatorname{Ln}\left[\frac{\pi}{4} \times \frac{m_{\mathrm{e}}}{m_{i}} \times \frac{q \times U_{\mathrm{a}}}{k \times T_{\mathrm{e}}}\right] \approx \\
& \approx-0,41 \text { Volt } \\
& \Rightarrow \phi_{\text {paroi }}=\phi_{\text {flottant }}-\Phi_{\text {flottant }} \approx-0,02 \text { Volt } \\
& \Rightarrow \phi_{\text {sonde }}=\varphi_{\text {sonde }}+\phi_{\text {paroi }} \approx-0,67 \text { Volt }
\end{aligned}
$$

Nous obtenons alors finalement pour la formule (12) :

$$
i_{\mathrm{il}} \approx n_{\mathrm{il}} \times 3,5 \times 10^{-20} \mathrm{~A}
$$

Nous pouvons maintenant calculer les courants moyens d'ions lents, et ceux des ions dérivants hors du sillage, pour les deux cas de mesure :

$$
\begin{gathered}
\left(i_{\mathrm{il}}\right)_{\max } \approx 2,1 \mathrm{nA} \text { et } i_{\mathrm{id}} \approx 45 \mathrm{nA} \\
\Rightarrow\left(\frac{i_{\mathrm{il}}}{i_{\mathrm{il}}+i_{\mathrm{id}}}\right)_{\max } \approx 4,5 \% \\
\left(i_{\mathrm{il}}\right)_{\min } \approx 0,16 \mathrm{nA} \text { et } i_{\mathrm{id}} \approx 48 \mathrm{nA} \\
\Rightarrow\left(\frac{i_{\mathrm{il}}}{i_{\mathrm{il}}+i_{\mathrm{id}}}\right)_{\text {min }} \approx 0,3 \% .
\end{gathered}
$$

2.3.3 Comparaison avec les expériences. - Nous avons exploité les deux sections de sillage proche réalisées à $(z / R \approx 5)$ où la contribution des ions lents est particulièrement sensible. On suppose comme les auteurs, et en accord avec nos résultats, que la contribution des ions lents est négligeable pour la pression la plus basse et que la distribution des ions dérivants est peu perturbée par la présence des ions lents, dans l'autre cas. On peut alors calculer dans ce dernier cas, la proportion d'ions lents dans le courant mesuré au centre du sillage. L'indice 0 indique une valeur hors du sillage, l'indice c une valeur au centre de celui-ci :

$$
\begin{aligned}
\max :\left(i_{\mathrm{il}}\right)_{0} & \approx\left(i_{\mathrm{il}}\right)_{\mathrm{c}}=i_{\mathrm{il}} \\
i_{0} & =i_{\mathrm{il}}+\left(i_{\mathrm{id}}\right)_{0} \\
i_{\mathrm{c}} & =i_{\mathrm{il}}+\left(i_{\mathrm{id}}\right)_{\mathrm{c}}
\end{aligned}
$$

$\min : i_{0} \approx\left(i_{\mathrm{id}}\right)_{0}$

$i_{\mathrm{c}} \approx\left(i_{\mathrm{id}}\right)_{\mathrm{c}}$

$$
\begin{aligned}
\left(\frac{\left(i_{\mathrm{id}}\right)_{\mathrm{c}}}{\left(i_{\mathrm{id}}\right)_{0}}\right)_{\max } \approx & \left(\frac{\left(i_{\mathrm{id}}\right)_{\mathrm{c}}}{\left(i_{\mathrm{id}}\right)_{0}}\right)_{\min } \approx\left(\frac{i_{\mathrm{c}}}{i_{0}}\right)_{\min } \\
\left(\frac{i_{\mathrm{c}}}{i_{0}}\right)_{\text {max }} & =\left(\frac{i_{\mathrm{i} 1}}{i_{0}}\right)_{\max }+\left(\frac{\left(i_{\mathrm{id}}\right)_{\mathrm{c}}}{i_{0}}\right)_{\max } \\
& \approx\left(\frac{i_{\mathrm{il}}}{i_{0}}\right)_{\text {max }}+\left(\frac{\left(i_{\mathrm{id}}\right)_{\mathrm{c}}}{\left(i_{\mathrm{id}}\right)_{0}}\right)_{\text {max }} \times \\
& \times\left(1-\frac{i_{\mathrm{il}}}{i_{0}}\right)_{\text {max }}
\end{aligned}
$$

d'où :

$$
\left(\frac{i_{\mathrm{il}}}{i_{0}}\right)_{\max } \approx \frac{\left(\frac{i_{\mathrm{c}}}{i_{0}}\right)_{\max }-\left(\frac{i_{\mathrm{c}}}{i_{0}}\right)_{\min }}{1-\left(\frac{i_{\mathrm{c}}}{i_{0}}\right)_{\min }}
$$

Numériquement on mesure (Fig. 12) :

$$
\left(\frac{i_{\mathrm{il}}}{i_{0}}\right)_{\max } \approx \frac{0,25-0,065}{1-0,065} \approx 19 \pm 2 \% \text {. }
$$

On observe donc une proportion environ quatre fois plus élevée que celle calculée analytiquement. Cette différence s'explique certainement par la distribution réelle non uniforme du plasma froid. Nous calculons en effet une densité moyenne qui est sûrement plus faible que la densité maximale atteinte au centre du faisceau de plasma dérivant. Par ailleurs, la diffusion ambipolaire des ions lents sous l'effet de la charge d'espace négative du sillage proche doit tendre à augmenter encore leur concentration au centre de celui-ci. Cette remarque est confirmée par l'observation d'un maximum central, imputable uniquement aux ions lents, dans la section la plus proche de la sphère, en $(z / R \approx 1)$ (Fig. 12). 
2.4 BILAN DE L'APPROCHE ANALYTIQUE. - Nous avons établi une expression conduisant à un ordre de grandeur satisfaisant de la densité d'ions lents, et éventuellement du courant qui en résulte sur une sonde polarisée négativement. Il est vraisemblable que de nouvelles expériences, réalisées dans des conditions adaptées à l'isolement spécifique de chaque variable, et tenant compte des sensibilités différentes mises en évidence pour chaque paramètre, permettraient d'affiner encore le calcul précédent. D'ores et déjà l'influence des principaux facteurs apparaît clairement, cependant les phénomènes physiques responsables du remplissage du sillage par les ions lents et consécutifs à leur collection par une surface négative, ne sont pas parfaitement clairs, comme en témoigne leur synthèse sous la forme d'un coefficient empirique (11) pour les expériences réalisées à PTS. L'approche suivante, à l'aide d'une simulation numérique directe du mouvement des particules, va nous permettre d'approfondir ces questions.

\section{Le code numérique.}

Nous avons utilisé comme point de départ, le code de simulation "PLASIM » (PLAsma SIMulation) élaboré par E. Coggiola et A. Soubeyran. Celui-ci modélise l'écoulement mésothermique d'un plasma sans collisions autour d'un cylindre infiniment long ou d'une sphère, présentant une petite section polarisée sur sa face aval. On trouvera une description précise des hypothèses et de la résolution numérique dans les références $[16,17,5]$. Ce code ne fait intervenir qu'une seule population ionique, animée d'une vitesse de dérive et généralement d'une agitation thermique beaucoup plus petite, en géométrie bidimensionnelle. Moyennant certaines simplifications, tel que l'absence de champ magnétique et le traitement implicite des électrons par l'approximation de Boltzman, il permet de déterminer le champ de potentiel et la distribution de densité ionique en aval de l'obstacle, où l'on observe un phénomène de sillage électrostatique. La figure 13 présente l'exemple d'une comparaison entre les mesures de Fournier et Pigache en l'absence d'ions lents, et la simulation numérique pertinente réalisée avec le code "PLASIM ». Les principales structures (cône de dépression et pic central de densité) sont correctement situées en position et amplitude, bien que subsistent de petites divergences (différence de l'angle d'ouverture du cône de dépression).

En raison de la grande différence de vitesse entre les ions dérivants et les ions lents, la détermination de la densité ionique en une seule étape, commune aux deux populations, n'est cependant pas possible dans ce code. Nous avons donc mis au point un programme spécifiquement destiné au calcul de la distribution de densité des ions lents, et l'avons

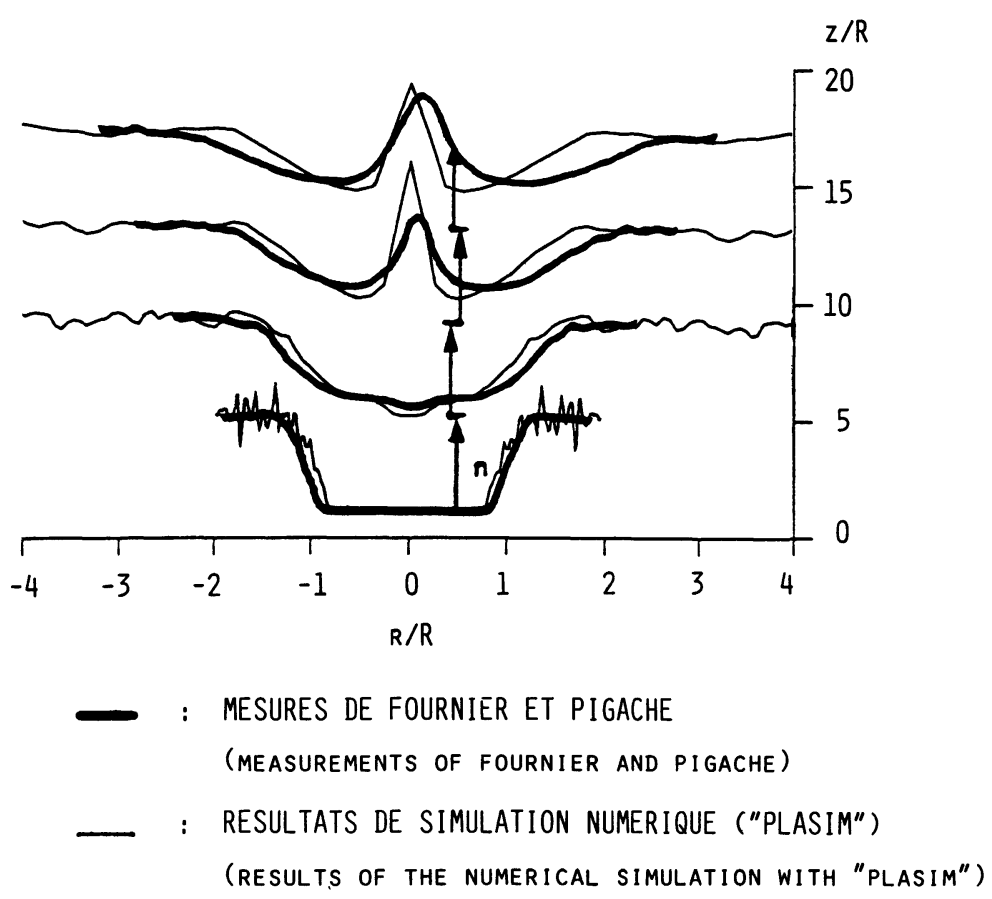

Fig. 13. - Comparaison entre les mesures de Fournier et Pigache et la simulation pertinente avec le code PLASIM. Les conditions d'interaction sont celles de la pression la plus basse de la figure 12, donc en l'absence d'ions lents.

[Comparison between Fournier and Pigache data and results from a numerical simulation with PLASIM, in the conditions of the lower pressure in figure 12 (no influence of slow ions)]. 
intégré dans un code plus général: "SMILE » (Simulation du Mouvement des Ions LEnts).

3.1 DENSITÉ DES IONS LENTS. - Dans un premier temps, le code «PLASIM » conduit à une distribution de densité ionique et de potentiel en l'absence d'ions lents. Les figures $14 \mathrm{a}$ et $\mathrm{b}$ (et suivantes) correspondent à la simulation d'une situation proche de celle de la chambre de PTS. Ces deux résultats nous servent alors pour calculer, d'une part la production, d'autre part les trajectoires des ions lents. Ceux-ci proviennent en effet d'un échange de charge supposé résonnant, qui conduit à un taux de production proportionnel à la densité d'ions rapides.

Numériquement, ce principe physique est utilisé par une méthode de Monte Carlo bidimensionnelle, où la probabilité de tirage est proportionnelle à la densité locale des ions dérivants, afin de générer des particules de simulation représentatives de la distribution d'un grand nombre d'ions lents réels. A chacune est alors affectée une vitesse thermique, de direction aléatoire et de module calculé, à partir d'un nombre aléatoire, de façon à représenter globalement une distribution maxwellienne réelle. Compte tenu des densités faibles que nous traitons, le déplacement de ces ions, comme celui des ions dérivants, peut être considéré comme sans collisions à l'échelle d'une chambre de simulation. Ainsi, en l'absence de gradients de potentiel (donc de champ électrostatique), ces ions se déplaceraient en ligne droite.
Or, l'observation de la distribution de potentiel calculée initialement (Fig. 14a) montre que seule une fraction du domaine de calcul présente des variations de potentiel significatives : le sillage situé à l'arrière du cylindre sur une largeur de quelques rayons de ce dernier. Afin d'alléger les calculs, nous avons donc décidé de ne "suivre" une particule, que dans le cas où sa trajectoire, initialement rectiligne, l'amène dans cette zone perturbée. Ainsi, nous ne calculons précisément la densité d'ions lents que dans cette région; partout ailleurs, le modèle analytique de diffusion simple établi précédemment (paragraphe 2) conduit à une densité moyenne uniforme en bon accord avec les résultats de la simulation numérique.

Contrairement au cas du code de simulation des ions dérivants, dans lequel les équations du mouvement sont résolues simultanément pour toutes les particules avec un pas de temps fixe, ici chaque ion est suivi individuellement avec un pas de temps adaptatif qui contribue à l'optimisation des calculs. Compte tenu des symétries du système et de la zone d'intérêt, le domaine de calcul est réduit à un quadrant, comme dans le code «PLASIM", mais les conditions aux limites sont choisies différemment, de façon à prendre en compte les ions lents provenant de toutes les parties du disque complet. Au centre et au bord extérieur, les particules sont absorbées, par contre elles sont réfléchies sur les parois latérales fictives en $\theta=0^{\circ}$ et $90^{\circ}$ (Fig. 15).

La méthode de calcul des densités à partir des

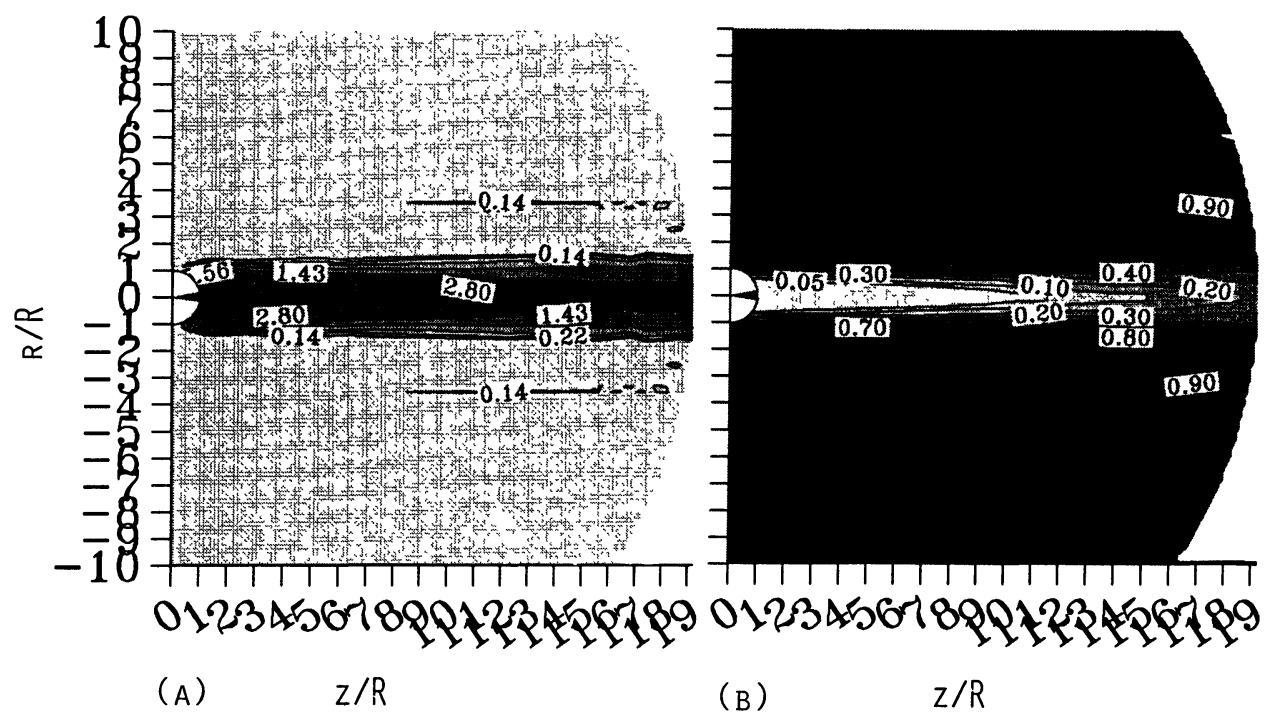

Fig. 14. - Distributions de potentiel (A) et de densité ionique (B) calculées par le code PLASIM, donc sans ions lents, dans des conditions correspondant à certaines mesures faites à PTS: $R=10 \mathrm{~cm} ; h \approx 4 \mathrm{~mm} ; U_{\mathrm{a}} \approx 60 \mathrm{eV}$; $F_{\mathrm{p}} \approx 5 \mathrm{MHz} ; \varphi \approx-20$ Volt. Le courant collecté sur la section polarisée est alors nul.

[Potential (A) and ionic density (B) distributions computed by the code PLASIM, without cold ions, with plasma parameters characteristic of the PTS experiment : $R=10 \mathrm{~cm} ; h \approx 4 \mathrm{~mm} ; U_{\mathrm{a}} \approx 60 \mathrm{eV} ; F_{\mathrm{p}} \approx 5 \mathrm{MHz} ; \varphi \approx-20 \mathrm{Volt}$. In this case no current is collected on the polarised rear surface.] 


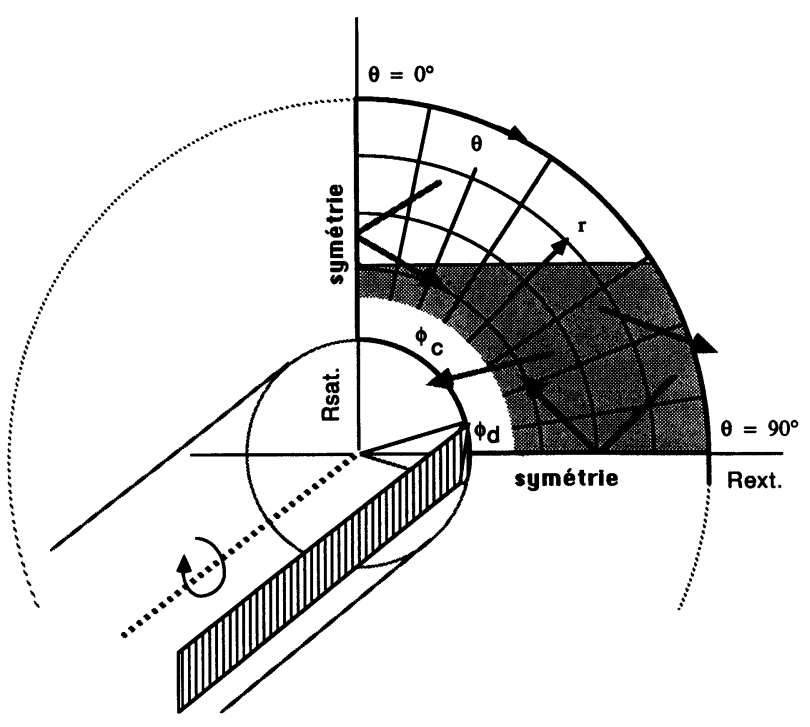

Fig. 15. - Représentation schématique de la zone d'interactions. La partie quadrillée correspond au domaine de calcul adopté, les conditions aux limites (flèches épaisses) et les coordonnées $(r, \theta)$ étant précisées. La surface arrière polarisée est hachurée. On a indiqué en grisé, la zone de suivi des particules. $\varphi_{\mathrm{c}}, \varphi_{\mathrm{d}}$ : potentiels à la surface du satellite; $R_{\text {sat }}$ : rayon du satellite, $R_{\text {ext }}$ : dimension caractéristique de la chambre de simulation.

[Schematic representation of the interaction area; the grided zone corresponds to the computational mesh, with indication of the coordinate system $(r, \theta)$ and of the boundary conditions (broad arrows). The rear polarised surface is hatched. The particles are followed in the shaded zone. $\varphi_{\mathrm{c}}, \varphi_{\mathrm{d}}$ : satellite surface potentials; $R_{\text {sat }}$ : satellite radius; $R_{\text {ext }}$ : characteristic dimension of the plasma chamber.]

trajectoires est analogue à celle utilisée dans « PLASIM » : à chaque pas de temps, une particule dépose sa charge autour de sa nouvelle position. Cette charge est distribuée entre les quatres nœuds du maillage les plus proches, selon une méthode de répartition bilinéaire dite "Cloud In Cell » [2], pondérée ici par le pas de temps variable. Lorsque la dernière particule a atteint une limite absorbante, la somme des charges accumulées en chaque nœud du réseau, le long de toutes les trajectoires suivies (quelques milliers), donne les densités ioniques en ces points, après une normalisation tenant compte en particulier du nombre total de particules générées, ainsi que des dimensions variables des cellules du réseau.

3.2 ITÉRATIONS. - Dans le cas où cette densité d'ions lents est petite devant celle des ions dérivants, la distribution de potentiel reste cohérente avec la nouvelle distribution de densité totale et ne nécessite donc aucun calcul supplémentaire. Ceci est vrai également si elle reste proportionnelle en tout point à la densité d'ions dérivants, auquel cas il ne s'agit que d'un changement de facteur de normalisation dans l'équation de Poisson.

Dans le cas général cependant, aucune de ces deux hypothèses n'est vérifiée, comme dans les expériences réalisées à PTS. Ainsi, il apparaît nécessaire de recalculer une nouvelle distribution de potentiel en accord avec la densité totale, par résolution de l'équation de Poisson. Cependant, dans ce nouveau champ de potentiel. les trajectoires ioniques ne seront plus les mêmes et une nouvelle distribution de densité ionique doit donc être aussi calculée. Ce processus doit ensuite être itéré jusqu'à l'obtention d'une cohérence physique des deux distributions (Figs. 16a à c). Toutes ces opérations et leur itération jusqu'à la convergence vers un état stationnaire, sont regroupées dans le nouveau code « SMILE », dont la figure 17 présente un algorithme simplifié.

3.3 RÉSultats. - Le premier résultat de cette simulation numérique est de confirmer quantitativement le calcul analytique précédent (paragraphe 2), par la mise en évidence d'une densité de plasma froid généralement plusieurs fois supérieure à celle du plasma dérivant, dans les conditions expérimentales adoptées par E. Coggiola dans la chambre de PTS. Les ions lents, concentrés au voisinage de la discontinuité de potentiel, modifient la gaine et provoquent un écrantage partiel du potentiel appliqué à l'arrière de la maquette cylindrique (Figs. 16a et c). Ainsi, les ions dérivants ne "voient " pratiquement plus le potentiel de la surface polarisée, et leur densité est donc analogue à celle résultant d'une simple expansion thermique dans le sillage (Fig. 18). Aucun courant ionique provenant du plasma dérivant ne peut être collecté par la paroi portée à quelques dizaines de volts négatifs dans le sillage, ainsi que nous l'avons montré auparavant $[16,17,5]$. Ce courant provient donc exclusivement des ions lents. Dans la simulation, il est supérieur à celui mesuré par E. Coggiola d'un facteur trois (Fig. 5). Les différences géométriques importantes entre les deux distributions de potentiel et les nombreuses approximations du modèle, n'autorisent probablement pas une meilleure précision. En effet, dans les expériences réalisées à PTS, le courant ionique était collecté par une petite plaque carrée sur la face aval du cylindre servant d'écran au plasma incident. La perturbation résultante était nettement tridimensionnelle, avec des équipotentielles approximativement hémisphériques, localisées au voisinage de la surface collectrice (Fig. 19a). Au contraire, dans la simulation numérique bidimensionnelle, toutes les équipotentielles s'étendent nécessairement suivant la longueur du cylindre et sont donc également plus développées dans la direction perpendiculaire (Fig. 19b). 


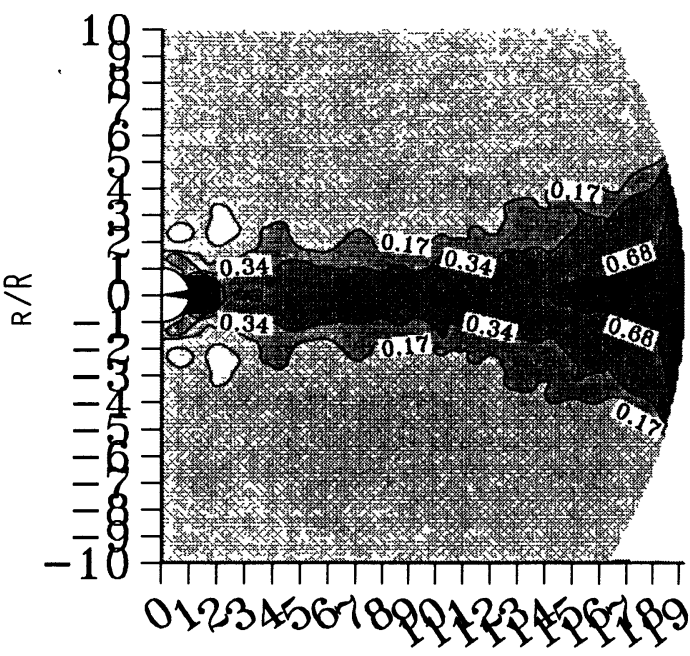

(A)
$\mathrm{Z} / \mathrm{R}$

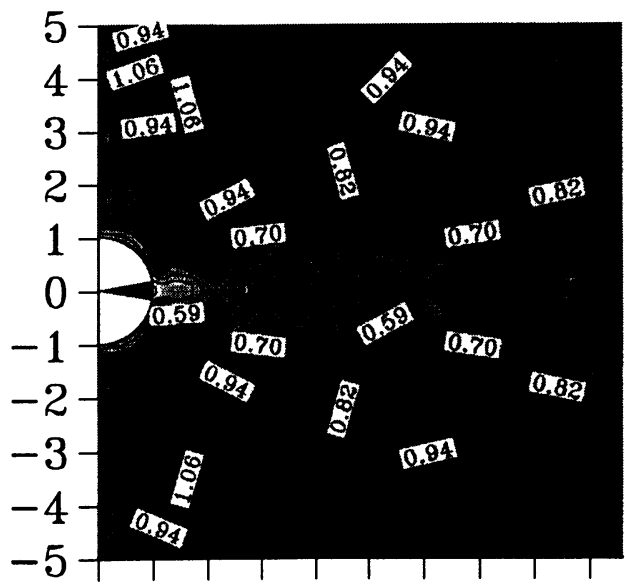

(c)

$z / R$

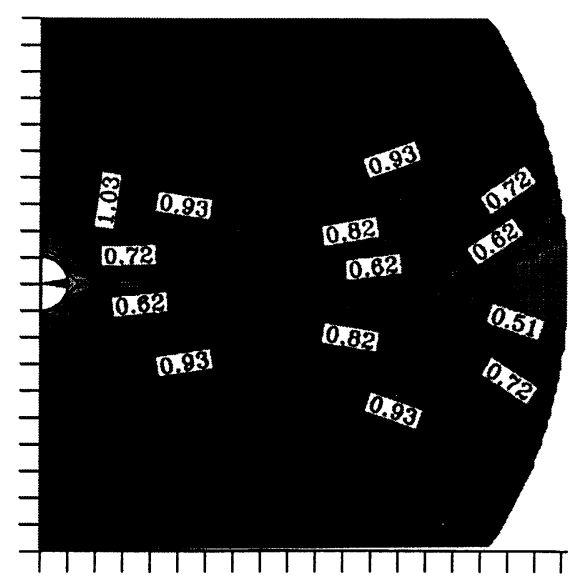

(E) $\quad Z / R$

Fig. 16. - Distributions de potentiel (A) et de densité (B et C (grossissement de la zone de gaine)) calculées par le code SMILE dans les mêmes conditions que la figure 14 , avec une pression de neutres: $P_{\mathrm{n}} \approx 5 \times 10^{-6}$ Torr $\approx$ $6,6 \times 10^{-4} \mathrm{~N} . \mathrm{m}^{-2}$. On constate le remplissage du sillage et l'adoucissement de toutes les structures, ainsi que l'écrantage du potentiel appliqué à l'arrière de la maquette.

[Potential (A) and density (B and C (zoom on the sheath region)) distributions in the wake of the cylinder, computed by the code SMILE for a pressure : $P_{\mathrm{n}} \approx 5 \times 10^{-6} \mathrm{Torr} \approx 6.6 \times 10^{-4} \mathrm{~N} \cdot \mathrm{m}^{-2}$. Filling of the wake and smoothing of all structures are obvious, as well as the screening of the rearward polarisation.]

Par ailleurs, nous avons simulé à l'aide du code numérique la diffusion simple, non collisionnelle, d'ions lents produits uniformément en l'absence de potentiels (Fig. 20), afin de comparer les résultats avec le calcul analytique. L'accord est satisfaisant :

* Le calcul du courant de diffusion collecté par le cylindre central conduit, par les deux approches, à des résultats analogues (écart relatif inférieur à $10 \%$ ).

* Le calcul analytique de la densité conduit à une valeur moyenne qui apparaît légèrement sous-estimée. Cependant, comme le montre la simulation numérique, la densité réelle d'ions lents n'est pas parfaitement uniforme du fait de l'absorption sur les parois cylindriques externe et interne.

\section{Conclusions.}

Afin de préciser les caractéristiques des ions lents dans une chambre de simulation du plasma ionosphérique, nous avons développé deux approches théoriques complémentaires que nous avons pu comparer à un ensemble de résultats expérimentaux variés. 


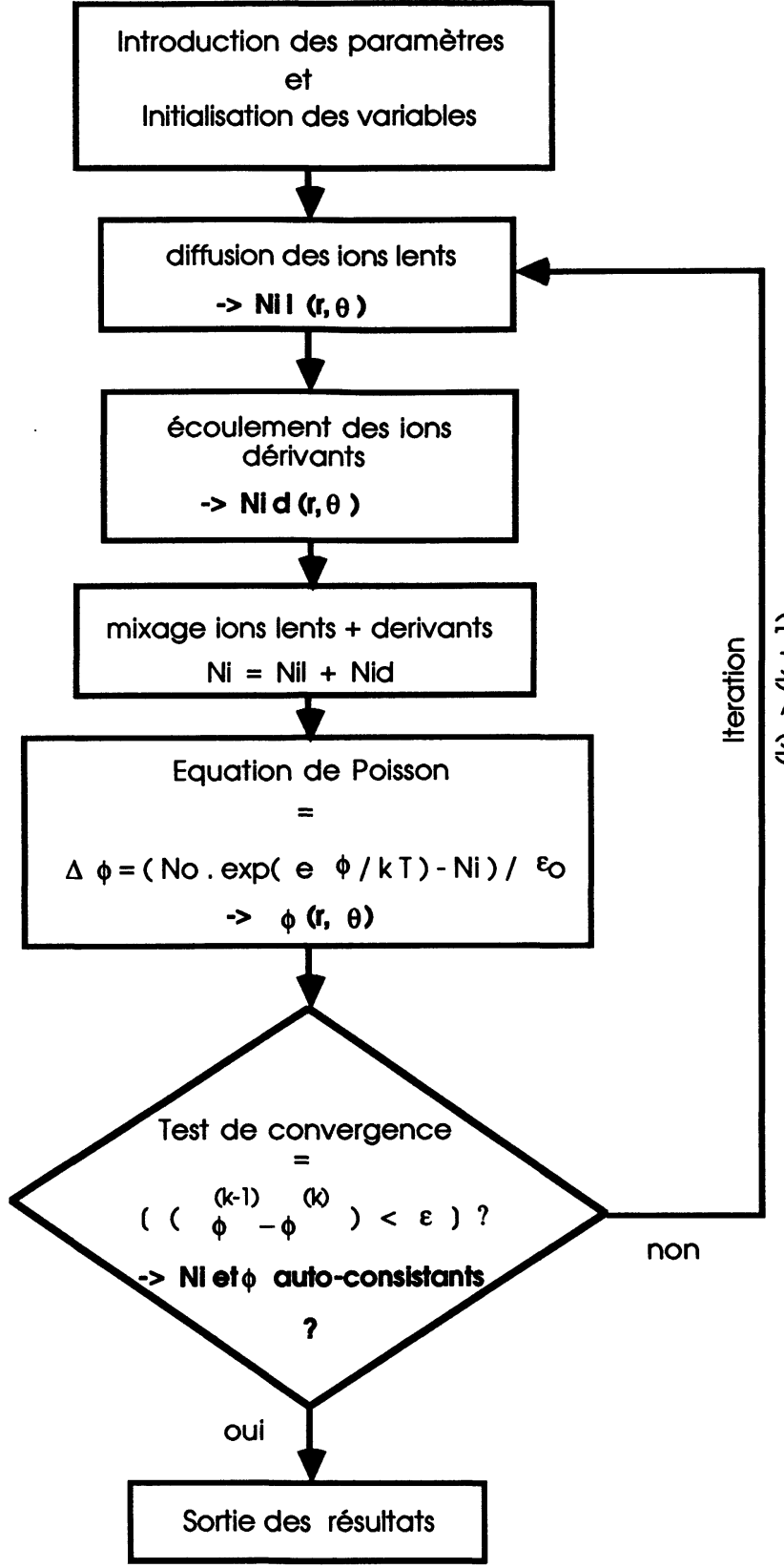

Fig. 17. - Algorithme simplifié du code SMILE : résolution numérique des équations couplées du mouvement, pour les ions lents d'une part et pour les ions dérivants d'autre part et de Poisson (explications dans le texte) : $N_{\text {il }}$ : densité des ions lents; $N_{\text {id }}$ : densité des ions dérivants $N$ : densité non erturbée à l'«infini »; $\Phi$ : potentiel.

[Algorithm of SMILE : numerical solution of the coupled motion and Poisson equations. The motion equations must be solved separatly for each class of ions: slow and drifting. $N_{\text {il }}$ : slow ions density; $N_{\text {id }}$ : drifting ions density ; $N_{0}$ : plasma density at infini ; $\Phi$ : potential.]

Un modèle analytique reliant entre eux les divers paramètres et variables pertinents, nous permet de rendre compte quantitativement des données expéri-

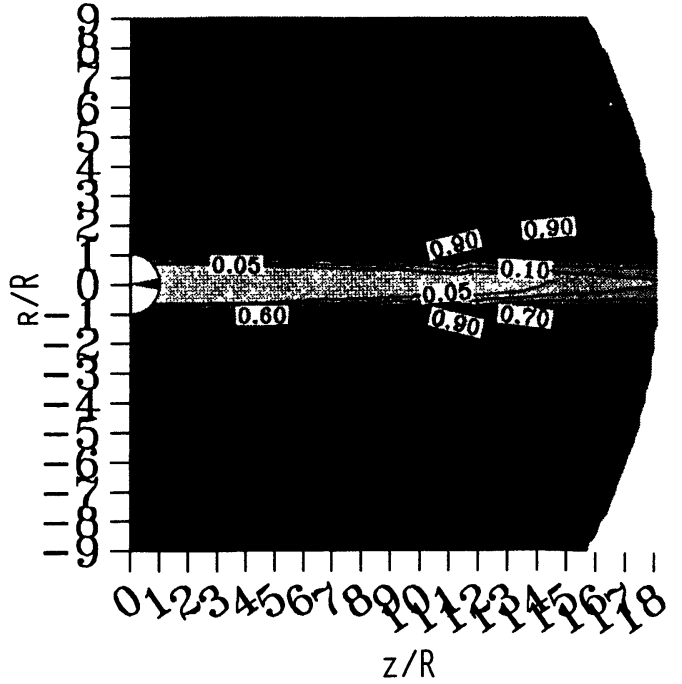

Fig. 18. - Distribution de densité des ions dérivants en l'absence de tout gradient de potentiel (situation fictive puisque l'équation de Poisson n'est alors pas vérifiée). La longueur du sillage, déterminée par l'agitation thermique, est maximale.

[Drifting ions density computed without any potential gradient (not actually possible because it does not verify the Poisson equation). The wake length depends only of the thermal movement and is maximum.]
(A) EXPERIENCEA ATS :3D

experımen :

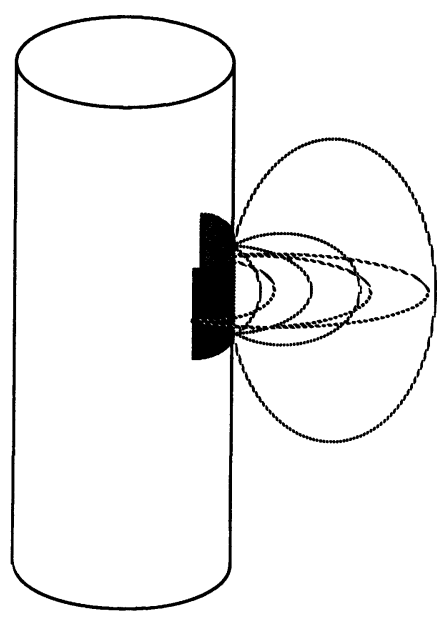

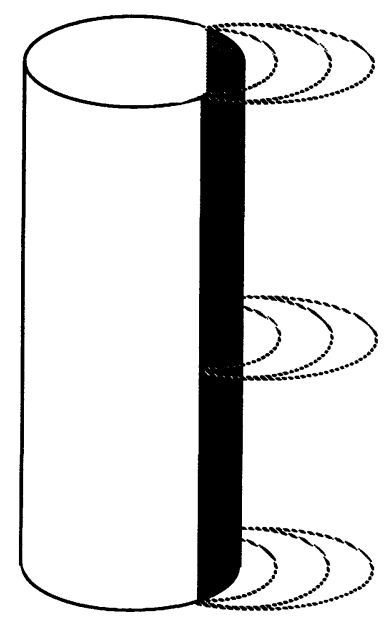

(B) SIMULATION NUMERIQUE :2D u 1 i uaion.
Fig. 19. - Comparaison entre la géométrie tridimensionnelle du potentiel autour de l'électrode de mesure dans les expériences de PTS, et la géométrie bidimensionnelle du potentiel calculé dans les codes PLASIM ou SMILE. La collection des ions lents n'est sûrement pas identique dans les deux cas.

[Comparison between the tridimensional potential around the electrode in the PTS experiments and the two-dimensional potential computed by PLASIM or SMILE. The slow ions may not be collected in the same way in the two cases.] 


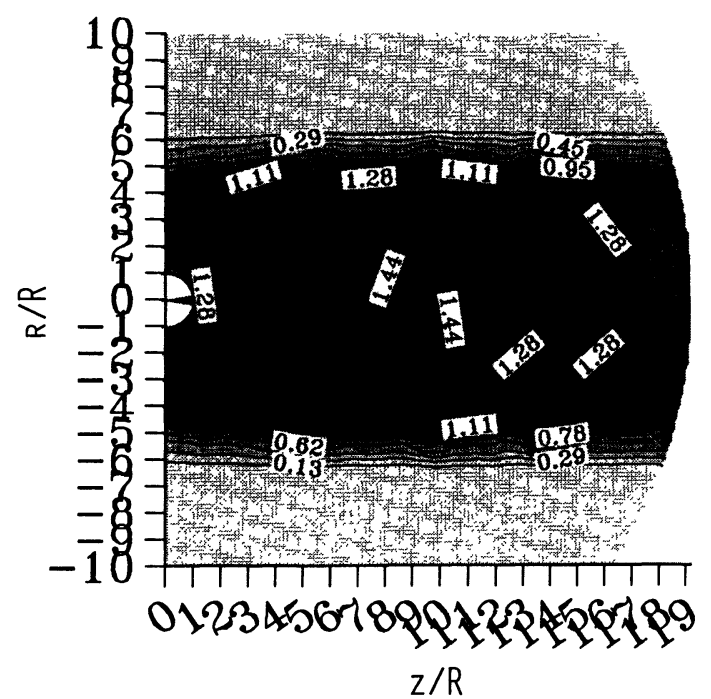

Fig. 20. - Distribution de densité des ions lents générés uniformément, calculée par SMILE en l'absence de gradient de potentiel. La distance à l'axe de suivi des particules est de $(5 . R)$ ce qui explique le calcul de la densité uniquement entre +5 et $-5 R$. Cette densité est normalisée par rapport au résultat du calcul analytique (paragraphe 2). On constate un bon accord puisque la moyenne n'est pas loin de 1 , mais la non-uniformité du plasma, due en particulier à la paroi externe, apparaît nettement.

[Slow ions density computed by SMILE from a uniformly generated plasma without potential gradient. The maximum distance from the centerline is $5 . R$, and the density is computed only in this region. This density is divided by the result of the analytical model, and we observe a reasonably good consistence (normalised densities around 1). The non uniformity caused by the walls is clearly evidenced.] mentales et en général, de leurs variations. Moyennant la détermination plus ou moins empirique de certains paramètres, nous pouvons en déduire l'importance des ions lents dans d'autres situations.

Un code de simulation numérique, contenant un minimum d'hypothèses physiques, dans des conditions géométriques simplifiées, nous permet de comprendre les phénomènes physiques importants, induits par les ions lents dans une expérimentation visant à reproduire les conditions d'orbite basse. Ceux-ci sont de deux ordres : d'une part la densité de cette population parasite modifie la densité totale et celle des ions dérivants n'est donc plus strictement analogue à celle de l'ionosphère, d'autre part l'isotropie et la faible énergie de la distribution de vitesse de ces ions lents, les rend très sensibles à toute différence de potentiel. C'est ainsi qu'ils viennent facilement remplir, au moins partiellement, le sillage électrostatique d'un objet et qu'ils neutralisent de même rapidement toute surface chargée négativement, quelle que soit son orientation.

Pour finir, remarquons que l'existence d'un plasma froid isotrope, autour de la navette spatiale fait aujourd'hui l'objet de controverses [11, 15]. Son origine serait vraisemblablement différente de celle du plasma en chambre expérimentale, mais il est tout à fait concevable que ses effets puissent être analogues, en tenant compte cependant de la présence du champ magnétique. $\mathrm{Si}$ cette existence venait à être confirmée et concernait aussi les autres grandes structures spatiales envisagées en orbite basse, elle pourrait bien jouer un rôle important dans la modération des différences de potentiels sur et au voisinage de ces objets.

\section{Bibliographie}

[1] Arnal Y., Simulation Expérimentale du Plasma Ionosphérique. Etude de Faisceaux de Plasmas et de Plasmas Uniformes dans une Structure Magnétique Multipolaire, Thèse de doctorat, Orléans (1977).

[2] Birdsall C. K. and Langdon A. B., Plasma Physics via Computer Simulation (Mc GrawHill, New York) 1985.

[3] Chapman B., Glow Discharge Processes, WileyInterscience Publication (1980) pp. 65-69.

[4] Clayden W. A. and Hurdle C. V., Diagnosis of a Plasma Beam Extracted from an Electron-bombardment Ion Source, in Plasmas and Interactions of Electromagnetic Radiation with Plasmas and Flames, AGARD CP, N 8, 2 (1965) 385.

[5] Coggiola E., Etude Théorique et Expérimentale de l'Ecoulement de Plasma autour d'un Cylindre Non Equipotentiel, Thèse doctorat ENSAE, CER Toulouse/DERTS, 16 juin 1988.
[6] Fournier G., Ecoulement de Plasma Sans Collisions Autour d'un Cylindre en Vue d'Applications aux Sondes Ionosphériques, Off. Nat. d'Etudes et de Rech. Aérosp., Châtillon, Publ. 137 (1971).

[7] Fournier G. and PIGACHE D., Wakes In Collisionless Plasma, Phys. Fluids 18 (1975) 1443-1453.

[8] Laframboise J. G. and Parker L. W., Probe Design for Orbit-Limited Current Collection, Phys. Fluids 16, N 5 (1973) 629-636.

[9] MARTIN A. R., Spacecraft-Plasma Interactions : Introduction and Review of Simulation Validity, City University Research Memo-Aero 73/1, 1973.

[10] Martin A. R. and Cox R. N., Simulation of Spacecraft Interactions with a Parallel-Streaming Plasma, Proceedings of 7th Space Simulation Conf., NASA SP-336 (1973) 215.

[11] Murphy G., Pickett J., D’Angelo N. and Kurth W. S., Measurements of Plasma Parameters in 
the vicinity of the Space Shuttle, Planetary Space Sci. 34 (1986) 993.

[12] Oran W. A., SAmir U. and Stone N. H., Slow Ions in Plasma Wind Tunnels, AIAA J. 14 (1976) 1141.

[13] Sajben M. and Blumenthal D. L., Experimental Study of a Rarefied Plasma Stream and its Interaction with Simple Bodies, AIAA Paper N 69-79 (1969).

[14] Salzborn E., Charge Exchange Cross Sections, IEEE Trans. Nucl. Sci. NS-23, N 2 (1976) 947958.

[15] Shu laï, Denig W. F., Murad E. and William J. Mc. Neil, The Role of Plasma Processes in the Space Shuttle Environment, Planet. Space Sci. 36, N 8 (1988) 841-849.

[16] Soubeyran A., Coggiola E. and Berthelier J. J., Simulation Numérique d'un Ecoulement de
Plasma en Aval d'une Sphère Non Equipotentielle, Revue Phys. Appl. 25 (1990) 395-404.

[17] Soubeyran A., LÉvy L. and Coggiola E., Numerical Simulation of the Wake on Non Equipotential Spacecrafts in the Ionosphere, Proceedings of the Spacecraft Charging Technology Conference 1989, Monterey, 31 oct.-3 nov. 1989 (à paraître).

[18] Stone N. H., The aerodynamics of Bodies in a Rarefied Ionized Gas with Applications to Spacecraft Environmental Dynamics, NASA Technical Paper № 1933 (1981).

[19] Stone N. H. and Rehman W. K., The Simulation Of Ionospheric Conditions For Space Vehicules, NASA TN D-5894 (1970).

[20] Wright K. H. Jr., A Study of Single and Binary Ion Plasma Expansion into Laboratory-generated Plasma Wakes, NASA-CR-4125 (1988). 